Article

\title{
An Efficient Parallel Extragradient Method for Systems of Variational Inequalities Involving Fixed Points of Demicontractive Mappings
}

\author{
Lateef Olakunle Jolaoso *(D) and Maggie Aphane \\ Department of Mathematics and Applied Mathematics, Sefako Makgatho Health Sciences University, \\ P.O. Box 94, Pretoria 0204, South Africa; maggie.aphane@smu.ac.za \\ * Correspondence: lateef.jolaoso@smu.ac.za \\ Received: 27 October 2020; Accepted: 18 November 2020; Published: 20 November 2020
}

\begin{abstract}
Herein, we present a new parallel extragradient method for solving systems of variational inequalities and common fixed point problems for demicontractive mappings in real Hilbert spaces. The algorithm determines the next iterate by computing a computationally inexpensive projection onto a sub-level set which is constructed using a convex combination of finite functions and an Armijo line-search procedure. A strong convergence result is proved without the need for the assumption of Lipschitz continuity on the cost operators of the variational inequalities. Finally, some numerical experiments are performed to illustrate the performance of the proposed method.
\end{abstract}

Keywords: extragradient method; variational inequalities; common solution; common fixed point; pseudomonotone; demicontractive

MSC: 65K15; 47J20; 65J15; 90C33

\section{Introduction}

Let $H$ be a real Hilbert space and $C$ be a nonempty, closed, and convex subset of $H$. Let $A: C \rightarrow H$ be an operator. The Variational Inequalities (VI) is defined as finding $x^{*} \in C$ such that

$$
\left\langle A x^{*}, y-x^{*}\right\rangle \geq 0, \quad \forall y \in C .
$$

The solution set of the VI (1) is denoted by $\operatorname{VI}(C, A)$. Mathematically, the VI is considered as a powerful tool for studying many nonlinear problems arising in mechanics, optimization, control network, equilibrium problems, etc.; see [1-3]. The problem of finding a common solution of a systems of VI has received a lot of attention by many authors recently, see, e.g., in [4-10] and references therein. This problem covers as special cases, convex feasibility problem, common equilibrium problem, etc. In this paper, we consider the following common problem.

Problem 1. Find an element $x^{*} \in C$ such that

$$
x^{*} \in\left(\bigcap_{i=1}^{N} V I\left(C, A_{i}\right)\right) \cap\left(\bigcap_{j=1}^{M} \operatorname{Fix}\left(T_{j}\right)\right),
$$

where for $i=1,2, \ldots, N, A_{i}: H \rightarrow H$ are pseudomonotonotone operators, $j=1,2, \ldots, M, T_{j}: H \rightarrow H$ are $k_{j}$-demicontractive mappings, $\operatorname{Fix}\left(T_{j}\right)=\left\{x \in H: T_{j} x=x\right\}$ denotes the fixed point set of $T_{j}$. 
The motivation for considering Problem 1 lies in its possible applications to mathematical models whose constraints can be expressed as the common variational inequalities and common fixed point problems. This happen in particular, in network resource allocations, image processing, Nash equilibrium problem, etc., see, e.g., in [11-14].

The simplest method for solving the VI (1) is the projection method of Goldstein [15] which is a natural extension of the gradient projection method, and for $x_{0} \in C, \lambda>0$ it is given by

$$
x_{n+1}=P_{C}\left(x_{n}-\lambda A x_{n}\right), \quad n \geq 0 .
$$

The projection method (3) converges weakly to a solution of VI (1) if and only if $A$ satisfies some strong conditions such as $\alpha$-strongly monotone and L-Lipschitz continuous. When this condition is relaxed, the method fails to convergence to any solution of the VI (1). Korpelevich [16] later introduced an Extragradient Method (EgM) for solving the VI when $A$ is monotone and L-Lipschitz continuous as follows, for $x_{0} \in C$,

$$
\left\{\begin{array}{l}
y_{n}=P_{C}\left(x_{n}-\lambda A x_{n}\right), \\
x_{n+1}=P_{C}\left(x_{n}-\lambda A y_{n}\right), \quad n \geq 0,
\end{array}\right.
$$

where $\lambda \in\left(0, \frac{1}{L}\right)$. The EgM has been extended to infinite-dimensional spaces by many authors, see, for instance, in [7,17-23]. More so, several modifications of the EgM have been introduced recently, see in [24-30]. For finding a common element in the set of solution of monotone variational inequalities and fixed point of $k$-demicontractive mapping, Mainge [14] introduced the following extragradient method, for $x_{0} \in C$,

$$
\left\{\begin{array}{l}
y_{n}=P_{C}\left(x_{n}-\lambda_{n} A x_{n}\right), \\
z_{n}=P_{C}\left(x_{n}-\lambda_{n} A y_{n}\right), \\
x_{n+1}=[(1-\alpha) I+\alpha T] u_{n}, \quad u_{n}=z_{n}-\gamma_{n} B z_{n}, \quad n \geq 0,
\end{array}\right.
$$

where $\left\{\lambda_{n}\right\},\left\{\gamma_{n}\right\} \subset(0, \infty), w \in[0,1], A: C \rightarrow H$ is a monotone and L-Lipschitz continuous, $T: H \rightarrow H$ is a $k$-demicontractive mapping and $B: H \rightarrow H$ is $\beta$-strongly monotone operator with $\beta>0$. The author proved a strong convergence for the sequence generated by (4) provided the step size $\lambda_{n}$ satisfies

$$
0<\liminf _{n \rightarrow \infty} \lambda_{n} \leq \limsup _{n \rightarrow \infty} \lambda_{n}<\frac{1}{L} .
$$

Recently, Hieu et al. [31] modified (4) and introduced the following extragradient method for approximating a common solution of VI and fixed point problem; given $x_{0} \in C$, compute $x_{n+1}$ via

$$
\left\{\begin{array}{l}
y_{n}=P_{C}\left(x_{n}-\lambda_{n} A x_{n}\right), \\
z_{n}=P_{C}\left(x_{n}-\rho_{n} A y_{n}\right), \\
w_{n}=P_{C}\left(x_{n}-\rho_{n} A z_{n}\right), \\
x_{n+1}=\left(1-\alpha_{n}\right) u_{n}+\alpha_{n} T u_{n}, \quad u_{n}=w_{n}-\gamma_{n} B w_{n}, \quad n \geq 0,
\end{array}\right.
$$

where $\left\{\rho_{n}\right\},\left\{\lambda_{n}\right\} \subset(0, \infty)$ such that $0 \leq \lambda_{n} \leq \rho_{n},\left\{\alpha_{n}\right\} \subset(0,1), A, T$ and $B$ are as defined for Algorithm (4). They also proved a strong convergence for the sequence generated by (6) with the aid of (5). An obvious disadvantage in (4) and (6) which impedes their wide usage is the assumption that the Lipschitz constant of $A$ admits a simple estimate. Moreover, in many practical problems, the cost operator may not satisfies Lipschitz condition.

On the other hand, for finding a common fixed point of quasi-nonexpansive mappings, Anh and Hieu [11,32] proposed a parallel hybrid algorithm as follows, 


$$
\left\{\begin{array}{l}
x_{0} \in C \\
y_{n}^{i}=\alpha_{n} x_{n}+\left(1-\alpha_{n}\right) T_{i} x_{n}, \quad i=1,2, \ldots, N \\
i_{n}=\operatorname{Argmax}\left\{\left\|y_{n}^{i}-x_{n}\right\|: i=1,2, \ldots, N\right\}, \quad \bar{y}_{n}:=y_{n}^{i_{n}}, \\
C_{n+1}=\left\{v \in C_{n}:\left\|v-\bar{y}_{n}\right\| \leq\left\|v-x_{n}\right\|\right\} \\
x_{n+1}=P_{C_{n+1}}\left(x_{0}\right) .
\end{array}\right.
$$

Furthermore, Censor et al. [6] proposed a parallel hybrid-extragradient method for finite family of variational inequalities as follows; choose $x_{0} \in H$, compute

$$
\left\{\begin{array}{l}
y_{n}^{i}=P_{C_{i}}\left(x_{n}-\lambda_{n}^{i} A_{i} x_{n}\right), \\
z_{n}^{i}=P_{C_{i}}\left(x_{n}-\lambda_{n}^{i} A_{i} y_{n}^{i}\right), \\
C_{n}^{i}=\left\{z \in H:\left\langle x_{n}-z_{n}^{i}, z-x_{n}-\gamma_{n}^{i}\left(z_{n}^{i}-x_{n}\right)\right\rangle \leq 0\right\}, \\
Q_{n}=\cap_{i=1}^{N} C_{n}^{i} \\
W_{n}=\left\{z \in H:\left\langle x_{0}-x_{n}, z-x_{n}\right\rangle \leq 0\right\}, \\
C_{n+1}=P_{Q_{n} \cap W_{n}} x_{0} .
\end{array}\right.
$$

Motivated by (7) and (8), Anh and Phuong [8] recently introduced the following Algorithm 1 parallel hybrid-extragradient method for solving variational inequalities and fixed point of nonexpansive mappings.

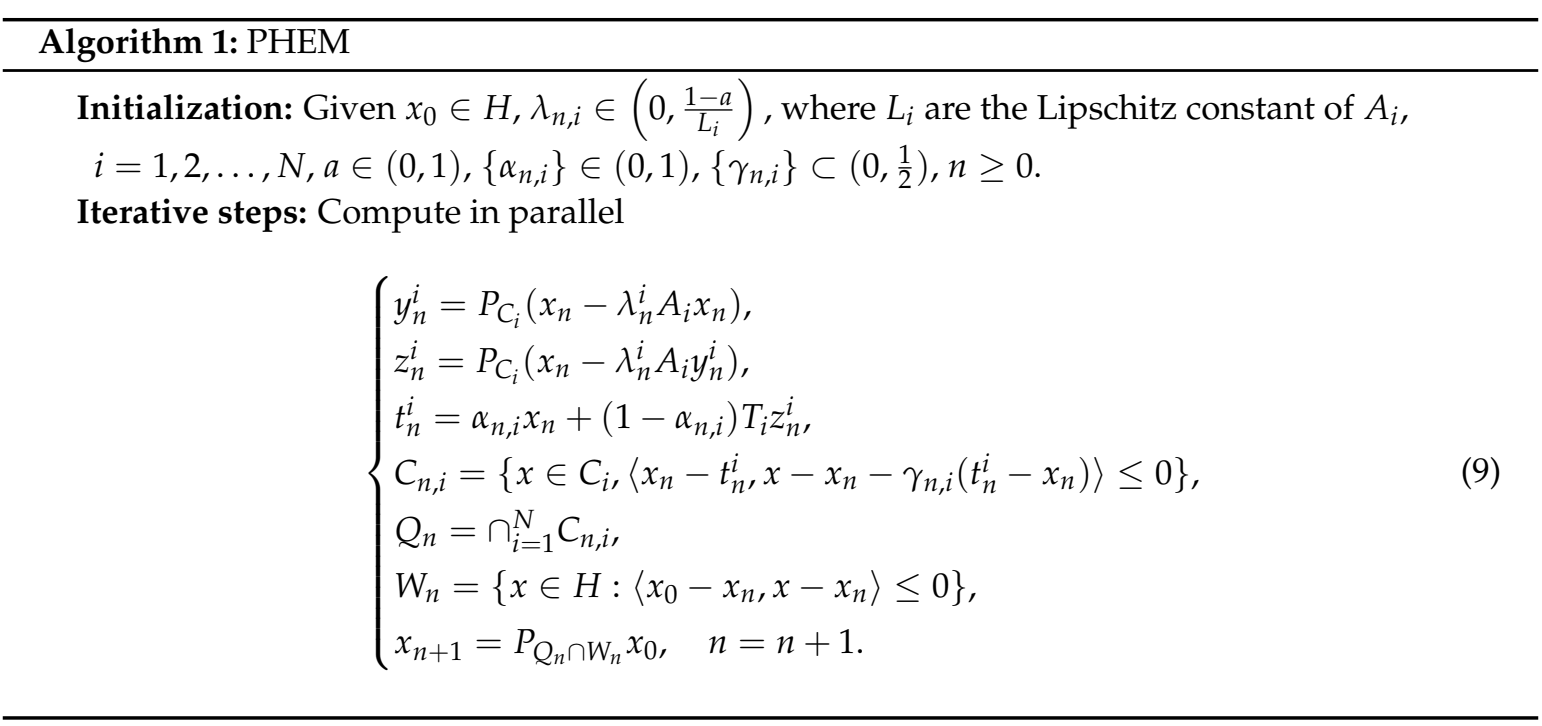

Meanwhile, Hieu [33] introduced a parallel hybrid-subgradient extragradient method which also required finding a farthest element from the iterate $x_{n}$ as follows.

The author proved that the sequence generated by Algorithm 2 converges strongly to a solution of the systems of VI. 


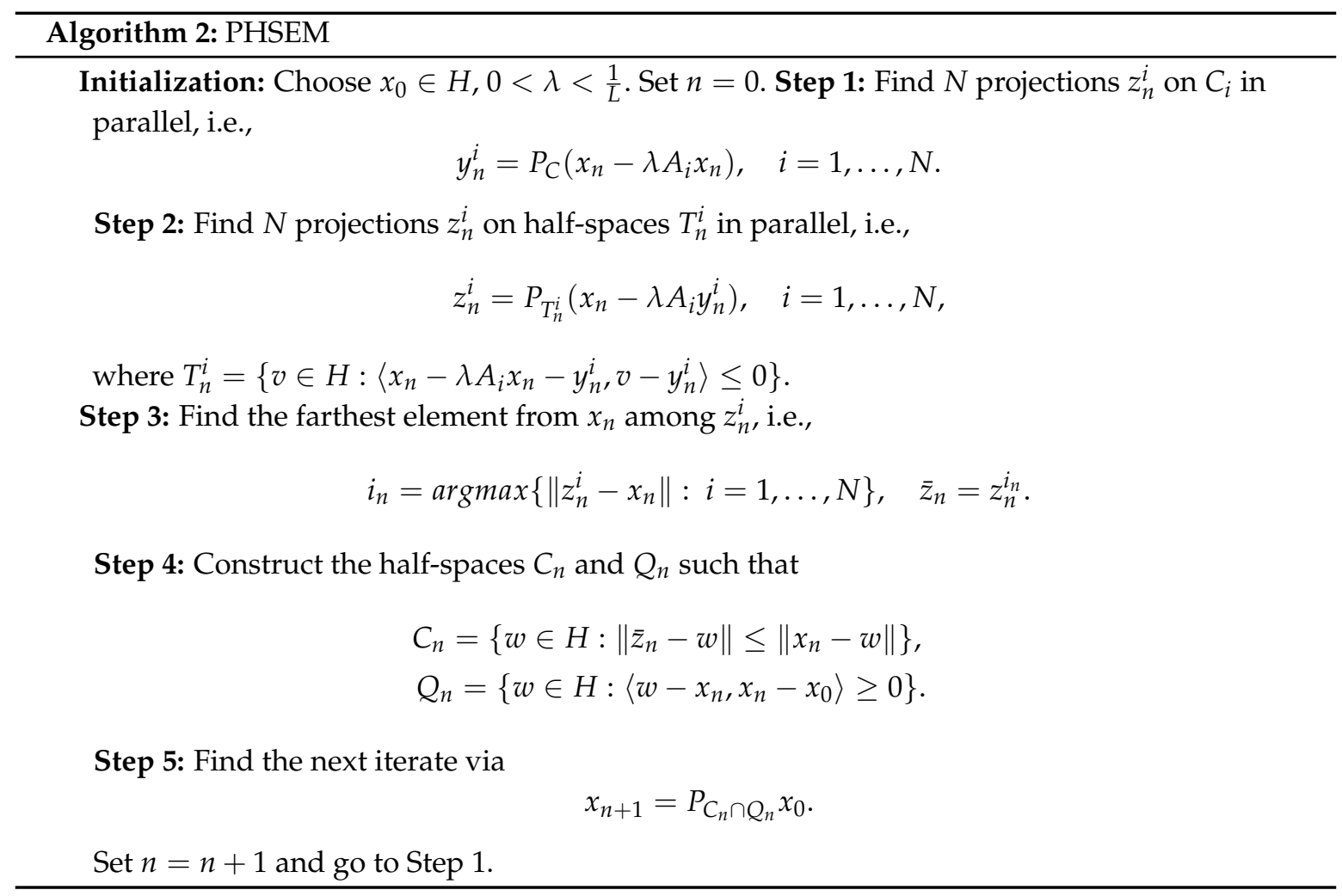

However, it should be observed that at each step in the parallel hybrid-extragradient methods mentioned above, one needs to calculate a projection onto the intersection of two sets $Q_{n}$ and $W_{n}$. This can be computationally expensive when the feasible set is not simple. Moreover, the convergence of the algorithms require prior knowledge of the Lipschitz constants of $A_{i}$ which are very difficult to estimate in practice.

Motivated by these results, in this paper, we introduce an efficient parallel-extragradient method which does not requires the computation of projection onto $Q_{n} \cap W_{n}$ and the prior estimates of the Lipschitz constants of $A_{i}$ for $i=1,2, \ldots, N$. In particular, we highlight some contributions in this paper as follows.

- In our method, the involved cost operators $A_{i}$ do not need to satisfy the Lipschitz condition. Instead, we assumed that $A_{i}$ are pseudomonotone and weakly sequentially continuous which is more general than the monotone and Lipschitz continuous used in previous results.

- The sequence generated by our method converges strongly to a solution of (2) without the aid of prior estimate of a Lipschitz constant.

- Furthermore, we performed only single projection onto $C$ in parallel and our algorithm does not need to find the farthest element from the iterate $x_{n}$.

- More so, our algorithm does not require the computation of projection onto $Q_{n} \cap W_{n}$ which make it easier for computations.

\section{Preliminaries}

In this section, we give some Definitions and basic results that will be used in our subsequent analysis. Let $H$ be a real Hilbert space. The weak and the strong convergence of $\left\{x_{n}\right\} \subset H$ to $x \in H$ is denoted by $x_{n} \rightarrow x$ and $x_{n} \rightarrow x$ as $n \rightarrow \infty$, respectively. Let $C$ be a nonempty, closed, and convex subset of $H$. The metric projection of $x \in H$ onto $C$ is defined as the necessary unique vector $P_{C}(x)$ satisfying

$$
\left\|x-P_{C} x\right\| \leq\|x-y\| \quad \forall y \in C .
$$


It is well known that $P_{C}$ has the following properties (see, e.g., in $[34,35]$ ).

(i) For each $x \in H$ and $z \in C$,

$$
z=P_{C} x \Leftrightarrow\langle x-z, z-y\rangle \geq 0, \quad \forall y \in C .
$$

(ii) For any $x, y \in H$,

$$
\left\langle P_{C} x-P_{C} y, x-y\right\rangle \geq\left\|P_{C} x-P_{C} y\right\|^{2}
$$

(iii) For any $x \in H$ and $y \in C$,

$$
\left\|P_{C} x-y\right\|^{2} \leq\|x-y\|^{2}-\left\|x-P_{C} x\right\|^{2} .
$$

Next, we state some classes of functions that play essential roles in our convergence analysis.

Definition 1. The operator $A: C \rightarrow H$ is said to be

1. $\beta$-strongly monotone if there exists $\beta>0$ such that

$$
\langle A x-A y, x-y\rangle \geq \beta\|x-y\| \quad \forall x, y \in C ;
$$

2. monotone if

$$
\langle A x-A y, x-y\rangle \geq 0 \quad \forall x, y \in C ;
$$

3. $\quad \eta$-strongly pseudomonotone if there exists $\eta>0$ such that

$$
\langle A x, y-x\rangle \geq 0 \Rightarrow\langle A y, y-x\rangle \geq \gamma\|x-y\|^{2},
$$

for all $x, y \in C$;

4. pseudomonotone if for all $x, y \in C$

$$
\langle A x, y-x\rangle \geq 0 \Rightarrow\langle A y, y-x\rangle \geq 0
$$

5. L-Lipschitz continuous if there exists a constant $L>0$ such that

$$
\|A x-A y\| \leq L\|x-y\|, \quad \forall x, y \in C .
$$

When $L \in(0,1)$, then $A$ is called a contraction;

6. weakly sequentially continuous if for any $\left\{x_{n}\right\} \subset H$ such that $x_{n} \rightarrow \bar{x}$ implies $A x_{n} \rightarrow A \bar{x}$.

It is easy to see that (1) $\Rightarrow(2) \Rightarrow(4)$ and (1) $\Rightarrow(3) \Rightarrow(4)$, but the converse implications do not hold in general, see, for instance, in [21].

Lemma 1 ([36] Lemma 2.1). Consider the VIP (1) with C being a nonempty closed convex subset of $H$ and $A: C \rightarrow H$ is pseudomonotone and continuous. Then, $\bar{x} \in \operatorname{VIP}(C, A)$ if and only if

$$
\langle A y, y-\bar{x}\rangle \geq 0 \quad \forall y \in C
$$

Definition 2 ([37]). A mapping $T: H \rightarrow H$ is called

(i) nonexpansive if

$$
\|T u-T v\| \leq\|u-v\|, \quad \forall u, v \in H ;
$$

(ii) quasi-nonexpansive mapping if $F(T) \neq \varnothing$ and

$$
\|T u-z\| \leq\|u-z\|, \quad \forall u \in H, z \in F(T) ;
$$


(iii) $\mu$-strictly pseudocontractive if there exists a constant $\mu \in[0,1)$ such that

$$
\|T u-T v\|^{2} \leq\|u-v\|^{2}+\mu\|(I-T) u-(I-T) v\|^{2} \quad \forall u, v \in H ;
$$

(iv) $\kappa$-demicontractive mapping if there exists $\kappa \in[0,1)$ and $F(T) \neq \varnothing$ such that

$$
\|T u-z\|^{2} \leq\|u-z\|^{2}+\kappa\|u-T u\|^{2}, \quad \forall u \in H, z \in F(T) .
$$

It is easy to see that the class of demicontractive mappings contains the class of quasi-nonexpansive and strictly pseudocontractive mappings. Due to this generality and its possible applications in applied analysis, the class of demicontractive mapping has continue to attracts the attention of many authors in this decade.

A bounded linear operator $A$ on $H$ is said to be strongly positive if there exists a constant $\gamma>0$ such that

$$
\langle x, A x\rangle \geq \gamma\|x\|^{2}, \quad \forall x \in H .
$$

It is known that when $A$ is strongly positive bounded linear operator with $0<\rho<\frac{1}{\|A\|}$, then

$$
\|I-\rho A\| \leq 1-\rho \gamma .
$$

For any real Hilbert space $H$, it is known that the following identities hold (see, e.g., in [38]).

Lemma 2. For all $x, y, z \in H$, then

(i) $\|x+y\|^{2}=\|x\|^{2}+2\langle x, y\rangle+\|y\|^{2}$,

(ii) $\|x+y\|^{2} \leq\|x\|^{2}+2\langle y, x+y\rangle$,

(iii) $\|\eta x+(1-\eta) y\|^{2}=\eta\|x\|^{2}+(1-\eta)\|y\|^{2}-\eta(1-\eta)\|x-y\|^{2}, \quad \forall \eta \in[0,1]$.

Lemma 3 ([24]). Let $C$ be a nonempty closed convex subset of a real Hilbert space $H$ and $h$ be a real-valued function on $H$. Suppose $D=\{x \in C: h(x) \leq 0\}$ is nonempty and $h$ is Lipschitz continuous on $C$ with modulus $\vartheta>0$, then

$$
\operatorname{dist}(x, D) \geq \vartheta^{-1} \max \{h(x), 0\} \quad \forall x \in C .
$$

Lemma 4 ([39]). Let $\left\{\Gamma_{n}\right\}$ be a non-negative real sequence satisfying $\Gamma_{n+1} \leq\left(1-\theta_{n}\right) \Gamma_{n}+\theta_{n} b_{n}$, where $\left\{\theta_{n}\right\} \subset(0,1), \sum_{n=0}^{\infty} \theta_{n}=\infty$ and $\left\{b_{n}\right\}$ is a sequence such that $\limsup _{n \rightarrow \infty} b_{n} \leq 0$. Then, $\lim _{n \rightarrow \infty} \Gamma_{n}=0$.

Lemma 5 ((Lemma 3.1) [37]). Let $\left\{a_{n}\right\}$ be a sequence of real numbers such that there exists a subsequence $\left\{a_{n_{i}}\right\}$ of $\left\{a_{n}\right\}$ with $a_{n_{i}}<a_{n_{i}+1}$ for all $i \in \mathbb{N}$. Consider the integer $\left\{m_{k}\right\}$ defined by

$$
m_{k}=\max \left\{j \leq k: a_{j}<a_{j+1}\right\} .
$$

Then $\left\{m_{k}\right\}$ is a non-decreasing sequence verifying $\lim _{n \rightarrow \infty} m_{n}=\infty$, and for all $k \in \mathbb{N}$, the following estimate holds,

$$
a_{m_{k}} \leq a_{m_{k}+1}, \quad \text { and } \quad a_{k} \leq a_{m_{k}+1}
$$

\section{Algorithm and Convergence Analysis}

In this section, we describe our algorithm and prove its convergence under suitable conditions. Let $H$ be a real Hilbert space and $C$ be a nonempty, closed, and convex subset of $H$. We suppose that the following assumptions hold. 


\section{Assumption 1.}

(A1) For $i=1,2, \ldots, N, A_{i}: H \rightarrow H$ are pseudomonotone, uniformly continuous and weakly sequentially continuous operators;

(A2) For $j=1,2, \ldots, M, T_{j}: H \rightarrow H$ are $\kappa_{j}$-demicontractive mappings with $\kappa=\max \left\{\kappa_{j}: 1 \leq j \leq M\right\}$ such that $I-T_{j}$ are demiclosed at zero;

(A3) $f: H \rightarrow H$ is an $\alpha$-contraction mapping with $\alpha \in(0,1)$;

(A4) For $k=1,2, \ldots, \bar{N}, B_{k}: H \rightarrow H$ are strongly positive bounded linear operators with coefficients $\gamma_{k}>0$, where $\bar{\gamma}=\min \left\{\gamma_{k}: 1 \leq k \leq \bar{N}\right\}$ and $0<\gamma<\frac{\bar{\gamma}}{2 \alpha}$;

(A5) The solution set

$$
\text { Sol }=\bigcap_{i=1}^{N} V I\left(C, A_{i}\right) \cap \bigcap_{j=1}^{M} \operatorname{Fix}\left(T_{j}\right)
$$

is nonempty.

We now present our method as follows.

Remark 1. Observe that we are at a solution of Problem (2) if $x_{n}=y_{n}=u_{n}$. We will implicitly assume that this does not occur after finitely many iterations so that our Algorithm 3 generates an infinitely sequence for our analysis.

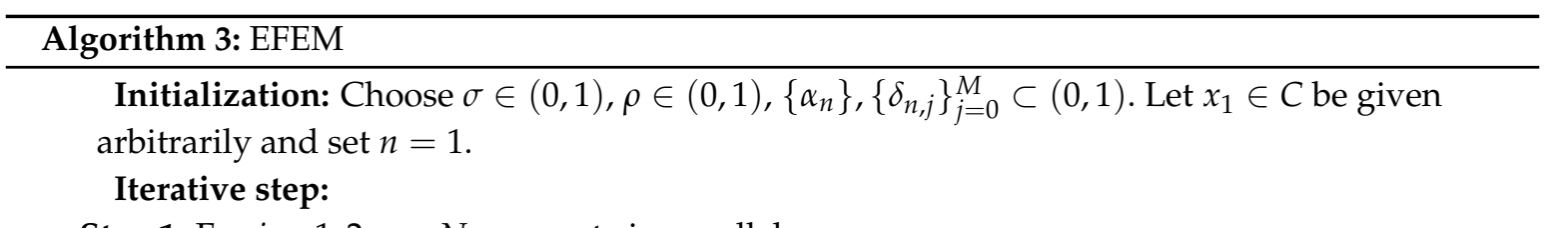

Step 1: For $i=1,2, \ldots, N$, compute in parallel

$$
y_{n}^{i}=P_{C}\left(x_{n}-A_{i} x_{n}\right)
$$

If $\theta^{i}\left(x_{n}\right)=x_{n}-y_{n}^{i}=0:$ set $x_{n}=w_{n}$ and do Step 3. Otherwise: do Step 2.

Step 2. Compute $z_{n}^{i}=x_{n}-\rho^{l_{n}} \theta^{i}\left(x_{n}\right)$, where $l_{n}$ is the smallest non-negative integer satisfying

$$
\left\langle A_{i} z_{n}^{i}, \theta^{i}\left(x_{n}\right)\right\rangle \geq \frac{\sigma}{2}\left\|\theta^{i}\left(x_{n}\right)\right\|^{2} .
$$

Set $w_{n}=P_{D_{n}}\left(x_{n}\right)$, where

$$
\begin{gathered}
D_{n}=\left\{x \in H: \sum_{i=1}^{N} \beta_{n}^{i} h_{n}^{i}\left(x_{n}\right) \leq 0\right\}, \\
\left\{\beta_{n}^{i}\right\}_{i=1}^{N} \subset(0,1) \text { such that } \sum_{i=1}^{N} \beta_{n}^{i}=1 \text {, and } h_{n}^{i}(x)=\left\langle A_{i} z_{n}^{i}, x-z_{n}^{i}\right\rangle .
\end{gathered}
$$

Step 3. Compute

$$
u_{n}=\delta_{n, 0} w_{n}+\sum_{n=1}^{M} \delta_{n, j} T_{j} w_{n}
$$

and

$$
x_{n+1}=\alpha_{n} \gamma f\left(x_{n}\right)+\left(I-\alpha_{n} \sum_{k=1}^{\bar{N}} c_{k} B_{k}\right) u_{n}
$$

where $\left\{c_{k}\right\}_{k=1}^{\bar{N}} \subset(0,1)$ such that $\sum_{k=1}^{\bar{N}} c_{k}=1$.

Stopping criterion: If $x_{n}=y_{n}=u_{n}$, then stop; otherwise, set $n:=n+1$ and go back to Step 1 . 
In order to prove the convergence of our algorithm, we assume that the control parameters satisfy the following conditions.

\section{Assumption 2.}

(B1) $\lim _{n \rightarrow \infty} \alpha_{n}=0$ and $\sum_{n=0}^{\infty} \alpha_{n}=+\infty$;

(B2) $\liminf _{n \rightarrow \infty}\left(\delta_{n, 0}-\kappa\right)>0$.

We begin the convergence analysis of Algorithm 3 by proving some useful Lemmas.

Lemma 6. Let $u^{*} \in$ Sol and $h_{n}^{i}$ be as defined in Algorithm 3. Then $h_{n}^{i}\left(x_{n}\right) \geq \rho^{l_{n}} \frac{\sigma}{2}\left\|x_{n}-y_{n}^{i}\right\|^{2}$ and $h_{n}^{i}\left(u^{*}\right) \leq 0$. In particular, if $\theta^{i}\left(x_{n}\right) \neq 0$, then $h_{n}^{i}\left(x_{n}\right)>0$ for all $n \in \mathbb{N}$.

Proof. As $z_{n}^{i}=x_{n}-\rho^{l_{n}}\left(x_{n}-y_{n}^{i}\right)$ for $i=1,2, \ldots, N$, then

$$
\begin{aligned}
h_{n}^{i}\left(x_{n}\right) & =\left\langle A z_{n}^{i}, x_{n}-z_{n}^{i}\right\rangle \\
& =\rho^{l_{n}}\left\langle A z_{n}^{i}, x_{n}-y_{n}^{i}\right\rangle \\
& \geq \rho^{l_{n}} \frac{\sigma}{2}\left\|x_{n}-y_{n}^{i}\right\|^{2} .
\end{aligned}
$$

Furthermore, if $x_{n} \neq y_{n}^{i}$ for $i=1,2, \ldots, N$, then $h_{n}^{i}\left(x_{n}\right)>0$. As $u^{*} \in$ Sol and $A_{i}$ are pseudomonotone, then

$$
\left\langle A y, y-u^{*}\right\rangle \geq 0 \quad \forall y \in C
$$

Therefore,

$$
\left\langle A z_{n}^{i}, z_{n}^{i}-u^{*}\right\rangle \geq 0
$$

Therefore,

$$
h_{n}^{i}\left(u^{*}\right)=\left\langle A z_{n}^{i}, u^{*}-z_{n}^{i}\right\rangle \leq 0 .
$$

Remark 2. Lemma 6 shows that $D_{n} \neq \varnothing$ and so $P_{D_{n}}$ is well defined. Consequently, Algorithm 3 is well defined.

Now we show that the sequence $\left\{x_{n}\right\}$ generated by Algorithm 3 is bounded.

Lemma 7. Let $\left\{x_{n}\right\}$ be the sequence generated by Algorithm 3. Then $\left\{x_{n}\right\}$ is bounded.

Proof. Let $u^{*} \in$ Sol, then from (11), we have

$$
\begin{aligned}
\left\|w_{n}-u^{*}\right\|^{2} & =\left\|P_{D_{n}} x_{n}-u^{*}\right\|^{2} \\
& \leq\left\|x_{n}-u^{*}\right\|^{2}-\left\|P_{D_{n}} x_{n}-x_{n}\right\|^{2} \\
& =\left\|x_{n}-u^{*}\right\|^{2}-\operatorname{dist}\left(x_{n}, D_{n}\right)^{2} \\
& \leq\left\|x_{n}-u^{*}\right\|^{2} .
\end{aligned}
$$


Moreover, from Lemma 2 (iii), we get

$$
\begin{aligned}
\left\|u_{n}-u^{*}\right\|^{2} & =\left\|\delta_{n, 0}\left(w_{n}-u^{*}\right)-\sum_{j=1}^{M} \delta_{n, j}\left(T_{j} w_{n}-u^{*}\right)\right\|^{2} \\
& =\delta_{n, 0}\left\|w_{n}-u^{*}\right\|^{2}+\sum_{j=1}^{M} \delta_{n, j}\left\|T_{j} w_{n}-u^{*}\right\|^{2}-\delta_{n, 0} \delta_{n, j}\left\|T_{j} w_{n}-w_{n}\right\|^{2} \\
& \left.\leq \delta_{n, 0}\left\|w_{n}-u^{*}\right\|^{2}+\sum_{j=1}^{M} \delta_{n, j}\left(\left\|w_{n}-u^{*}\right\|^{2}+\kappa_{j}\left\|w_{n}-T_{j} w_{n}\right\|^{2}\right)\right)-\delta_{n, 0} \delta_{n, j}\left\|T_{j} w_{n}-w_{n}\right\|^{2} \\
& \leq\left\|w_{n}-u^{*}\right\|^{2}-\sum_{j=1}^{M}\left(\delta_{n, 0}-\kappa\right) \delta_{n, j}\left\|w_{n}-T_{j} w_{n}\right\|^{2} \\
& \leq\left\|x_{n}-u^{*}\right\|^{2}-\sum_{j=1}^{M}\left(\delta_{n, 0}-\kappa\right) \delta_{n, j}\left\|w_{n}-T_{j} w_{n}\right\|^{2} .
\end{aligned}
$$

This implies that

$$
\left\|u_{n}-u^{*}\right\| \leq\left\|x_{n}-u^{*}\right\| .
$$

Then from (13), we obtain

$$
\begin{aligned}
\left\|x_{n+1}-u^{*}\right\| & =\left\|\alpha_{n} \gamma f\left(x_{n}\right)+\left(1-\alpha_{n} \sum_{k=0}^{\bar{N}} c_{k} B_{k}\right) u_{n}-u^{*}\right\| \\
& =\left\|\alpha_{n}\left(\gamma f\left(x_{n}\right)-\sum_{k=0} c_{k} B_{k} u^{*}\right)+\left(I-\alpha_{n} \sum_{k=0}^{\bar{N}} c_{k} B_{k}\right) u_{k}-\left(I-\alpha_{n} \sum_{k=0}^{\bar{N}} c_{k} B_{k}\right) u^{*}\right\| \\
& \leq\left\|\left(I-\alpha_{n} \sum_{k=0}^{\bar{N}} c_{k} B_{k}\right)\left(u_{n}-u^{*}\right)\right\|+\alpha_{n} \gamma\left\|f\left(x_{n}\right)-f\left(u^{*}\right)\right\|+\alpha_{n}\left\|\gamma f\left(u^{*}\right)-\sum_{k=0}^{\bar{N}} c_{k} B_{k} u^{*}\right\| \\
& \leq\left(1-\alpha_{n} \sum_{k=0}^{\bar{N}} c_{k} \gamma_{k}\right)\left\|u_{n}-u^{*}\right\|+\alpha_{n} \alpha \gamma\left\|x_{n}-u^{*}\right\|+\alpha_{n}\left\|\gamma f\left(u^{*}\right)-\sum_{k=0}^{\bar{N}} c_{k} B_{k} u^{*}\right\| \\
& \leq\left(1-\alpha_{n} \sum_{k=0}^{\bar{N}} c_{k} \bar{\gamma}\right)\left\|x_{n}-u^{*}\right\|+\alpha_{n} \alpha \gamma\left\|x_{n}-u^{*}\right\|+\alpha_{n}\left\|\gamma f\left(u^{*}\right)-\sum_{k=0}^{\bar{N}} c_{k} B_{k} u^{*}\right\| \\
& =\left(1-\alpha_{n} \bar{\gamma}\right)\left\|x_{n}-u^{*}\right\|+\alpha_{n} \alpha \gamma\left\|x_{n}-u^{*}\right\|+\alpha_{n}\left\|\gamma f\left(u^{*}\right)-\sum_{k=0}^{\bar{N}} c_{k} B_{k} u^{*}\right\| \\
& =\left(1-\alpha_{n}(\bar{\gamma}-\alpha \gamma)\left\|x_{n}-u^{*}\right\|+\alpha_{n}(\bar{\gamma}-\alpha \gamma) \frac{\left\|\gamma f\left(u^{*}\right)-\sum_{k=0}^{\bar{N}} c_{k} B_{k} u^{*}\right\|}{(\bar{\gamma}-\alpha \gamma)}\right. \\
& \leq \max \left\{\left\|x_{n}-u^{*}\right\|, \frac{\left\|\gamma f\left(u^{*}\right)-\sum_{k=0}^{\bar{N}} c_{k} B_{k} u^{*}\right\|}{(\bar{\gamma}-\alpha \gamma)}\right\} .
\end{aligned}
$$

By induction, we have

$$
\left\|x_{n}-u^{*}\right\| \leq \max \left\{\left\|x_{1}-u^{*}\right\|, \frac{\left\|\gamma f\left(u^{*}\right)-\sum_{k=0}^{\bar{N}} c_{k} B_{k} u^{*}\right\|}{(\bar{\gamma}-\alpha \gamma)}\right\} .
$$

This implies that $\left\{x_{n}\right\}$ is bounded.

Lemma 8. Let $u^{*} \in$ Sol and $\left\{x_{n}\right\}$ be the sequence generated by Algorithm 3, then $\left\{x_{n}\right\}$ satisfies the following estimates.

(i)

$$
\left\|w_{n}-u^{*}\right\|^{2} \leq\left\|x_{n}-u^{*}\right\|^{2}-\left(\frac{\sigma \rho^{l_{n}}}{L} \sum_{i=1}^{N} \beta_{n}^{i}\left\|x_{n}-y_{n}^{i}\right\|^{2}\right)
$$


(ii)

for some $L \geq 0$

$$
s_{n+1} \leq\left(1-a_{n}\right) s_{n}+a_{n} b_{n}
$$

where

$$
\begin{aligned}
& s_{n}=\left\|x_{n}-u^{*}\right\|^{2}, \quad a_{n}=\frac{2 \alpha_{n}(\bar{\gamma}-\alpha \gamma)}{1-\alpha_{n} \alpha \gamma}, \\
& b_{n}=\alpha_{n} M+\frac{\left\langle\gamma f\left(u^{*}\right)-\sum_{k=0}^{\bar{N}} c_{k} B_{k} u^{*}, x_{n+1}-u^{*}\right\rangle}{\bar{\gamma}-\alpha_{n} \gamma},
\end{aligned}
$$

for some $M>0$.

Proof. As $\left\{x_{n}\right\}$ is bounded and $A_{i}$ are continuous on bounded subsets of $H$, then $\left\{A_{i} x_{n}\right\}$ are bounded, and thus there exists some constants $L i>0$ such that

$$
\left\|A_{i} x_{n}\right\| \leq \frac{L_{i}}{2} \quad \forall n \in \mathbb{N}, i=1,2, \ldots, N .
$$

Consequently,

$$
\left\|A_{i} x_{n}\right\| \leq \frac{L}{2} \quad \text { where } \quad L=\max \left\{L_{i}, i=1,2, \ldots, N\right\} .
$$

Therefore from Lemma 3, we have

$$
\operatorname{dist}\left(x_{n}, D_{n}\right) \geq \frac{2}{L} \sum_{i=1}^{N} \beta_{n}^{i} h_{n}^{i}\left(x_{n}\right), \quad \forall n \geq 0 .
$$

Thus from (16) and (18), we get

$$
\begin{aligned}
\left\|w_{n}-u^{*}\right\|^{2} & =\left\|x_{n}-u^{*}\right\|^{2}-\operatorname{dist}\left(x_{n}, D_{n}\right)^{2} \\
& \leq\left\|w_{n}-u^{*}\right\|^{2}-\left(\frac{2}{L} \sum_{i=1}^{N} \beta_{n}^{i} h_{n}^{i}\left(x_{n}\right)\right)^{2} .
\end{aligned}
$$

Hence from Lemma 6, we obtain

$$
\left\|w_{n}-u^{*}\right\|^{2} \leq\left\|x_{n}-u^{*}\right\|^{2}-\left(\frac{\sigma \rho^{l_{n}}}{L} \sum_{i=1}^{N} \beta_{n}^{i}\left\|x_{n}-y_{n}^{i}\right\|^{2}\right) .
$$

This established (i).

Moreover, we have from Algorithm 3 that 


$$
\begin{aligned}
\left\|x_{n+1}-u^{*}\right\|^{2}= & \left\|\alpha_{n} \gamma f\left(x_{n}\right)+\left(I-\alpha_{n} \sum_{k=0}^{\bar{N}} c_{k} B_{k}\right) u_{n}-u^{*}\right\|^{2} \\
= & \left\|\alpha_{n}\left(\gamma f\left(x_{n}\right)-\sum_{k=0}^{\bar{N}} c_{k} B_{k} u^{*}\right)+\left(1-\alpha_{n} \sum_{k=0}^{\bar{N}} c_{k} B_{k}\right) u_{n}-\left(1-\alpha_{n} \sum_{k=0}^{\bar{N}} c_{k} B_{k}\right) u^{*}\right\|^{2} \\
\leq & \left\|\left(1-\alpha_{n} \sum_{k=0}^{\bar{N}} c_{k} B_{k}\right) u_{n}-\left(1-\alpha_{n} \sum_{k=0}^{\bar{N}} c_{k} B_{k}\right) u^{*}\right\|^{2} \\
& +2 \alpha_{n}\left\langle\gamma f\left(x_{n}\right)-\sum_{k=0}^{\bar{N}} c_{k} B_{k} u^{*}, x_{n+1}-u^{*}\right\rangle \\
\leq & \left(1-\alpha_{n} \sum_{k=0} c_{k} \gamma\right)^{2}\left\|u_{n}-u^{*}\right\|^{2}+2 \alpha_{n} \gamma\left\langle f\left(x_{n}\right)-f\left(u^{*}\right), x_{n+1}-u^{*}\right\rangle \\
& +2 \alpha_{n}\left\langle\gamma f\left(u^{*}\right)-\sum_{k=0}^{\bar{N}} c_{k} B_{k} u^{*}, x_{n+1}-u^{*}\right\rangle \\
\leq & \left(1-\alpha_{n} \sum_{k=0}^{\bar{N}} c_{k} \bar{\gamma}\right)^{2}\left\|x_{n}-u^{*}\right\|^{2}+2 \alpha_{n} \alpha \gamma\left\|x_{n}-u^{*}\right\|\left\|x_{n+1}-u^{*}\right\| \\
& +2 \alpha_{n}\left\langle\gamma f\left(u^{*}\right)-\sum_{k=0}^{\bar{N}} c_{k} B_{k} u^{*}, x_{n+1}-u^{*}\right\rangle \\
\leq & \left(1-\alpha_{n} \bar{\gamma}\right)^{2}\left\|x_{n}-u^{*}\right\|^{2}+\alpha_{n} \gamma\left(\left\|x_{n}-u^{*}\right\|^{2}+\left\|x_{n+1}-u^{*}\right\|^{2}\right) \\
& +2 \alpha_{n}\left\langle\gamma f\left(u^{*}\right)-\sum_{k=0}^{N} c_{k} B_{k} u^{*}, x_{n+1}-u^{*}\right\rangle .
\end{aligned}
$$

Therefore, we obtain

$$
\begin{aligned}
\left\|x_{n+1}-u^{*}\right\|^{2} \leq & \frac{\left(1-\alpha_{n} \bar{\gamma}\right)^{2}+\alpha_{n} \alpha \gamma}{1-\alpha_{n} \alpha \gamma}\left\|x_{n}-u^{*}\right\|^{2}+\frac{2 \alpha_{n}}{1-\alpha_{n} \alpha \gamma}\left\langle\gamma f\left(u^{*}\right)-\sum_{k=0}^{\bar{N}} c_{k} B_{k} u^{*}, x_{n+1}-u^{*}\right\rangle \\
= & \left(1-\frac{2 \alpha_{n}(\bar{\gamma}-\alpha \gamma)}{1-\alpha_{n} \alpha \gamma}\right)\left\|x_{n}-u^{*}\right\|^{2}+\frac{\alpha_{n}^{2} \bar{\gamma}^{2}}{2-\alpha_{n} \alpha \gamma}\left\|x_{n}-u^{*}\right\|^{2} \\
& +\frac{2 \alpha_{n}}{1-\alpha_{n} \alpha \gamma}\left\langle\gamma f\left(u^{*}\right)-\sum_{k=0}^{\bar{N}} c_{k} B_{k} u^{*}, x_{n+1}-u^{*}\right\rangle \\
= & \left(1-\frac{2 \alpha_{n}(\bar{\gamma}-\alpha \gamma)}{1-\alpha_{n} \alpha \gamma}\right)\left\|x_{n}-u^{*}\right\|^{2} \\
& +\frac{2 \alpha_{n}(\bar{\gamma}-\alpha \gamma)}{1-\alpha_{n} \alpha \gamma}\left(\alpha_{n} M+\frac{\left\langle\gamma f\left(u^{*}\right)-\sum_{k=0}^{\bar{N}} c_{k} B_{k} u^{*}, x_{n+1}-u^{*}\right\rangle}{\bar{\gamma}-\alpha_{n} \gamma}\right) \\
= & \left(1-a_{n}\right) s_{n}+a_{n} b_{n},
\end{aligned}
$$

where the existence of $M$ follows from the boundedness of $\left\{x_{n}\right\}$. This completes the proof.

Lemma 9. Let $\left\{x_{n_{l}}\right\}$ be a subsequence of $\left\{x_{n}\right\}$ generated by Algorithm 3 such that $\left\{x_{n_{l}}\right\}$ converges weakly to $p \in H$ and $\lim _{l \rightarrow \infty}\left\|x_{n_{l}}-y_{n_{l}}^{i}\right\|=0$, for all $i=1,2, \ldots, N$. Then

(i) $0 \leq \liminf _{l \rightarrow \infty}\left\langle A_{i} x_{n_{l}}, x-x_{n_{l}}\right\rangle$, for all $x \in C, i=1,2, \ldots, N$;

(ii) $p \in \bigcap_{i=1}^{N} \operatorname{VI}\left(C, A_{i}\right)$.

Proof. (i) From the Definition of $y_{n}^{i}$ and (10), we have

$$
\left\langle x_{n_{l}}-A_{i} x_{n_{l}}-y_{n_{l}}^{i}, x-y_{n_{l}}^{i}\right\rangle \leq 0, \quad \forall x \in C, i=1,2, \ldots, N .
$$


Thus,

$$
\left\langle x_{n_{l}}-y_{n_{l}}^{i}, x-y_{n_{l}}^{i}\right\rangle \leq\left\langle A_{i} x_{n_{l}}, x-y_{n_{l}}^{i}\right\rangle, \quad \forall x \in C, i=1,2 \ldots, N .
$$

This implies that

$$
\left\langle x_{n_{l}}-y_{n_{l}}^{i}, x-y_{n_{l}}\right\rangle+\left\langle A_{i} x_{n_{l}}, y_{n_{l}}^{i}-x_{n_{l}}\right\rangle \leq\left\langle A x_{n_{l}}, x-x_{n_{l}}\right\rangle, \quad \forall x \in C, i=1,2, \ldots, N .
$$

Fix $x \in C$ and let $l \rightarrow \infty$ in (19), since $\left\|y_{n_{l}}^{i}-x_{n_{l}}\right\| \rightarrow 0$, then

$$
0 \leq \liminf _{l \rightarrow \infty}\left\langle A_{i} x_{n_{l}}, x-x_{n_{l}}\right\rangle, \quad \forall x \in C, i=1,2, \ldots, N .
$$

(ii) Suppose $\left\{\xi_{l}\right\}$ is a decreasing sequence of non-negative numbers such that $\xi_{l} \rightarrow 0$ as $l \rightarrow \infty$. For each $\xi_{l}$, we denote by $N_{l}$ the smallest positive integer such that

$$
\left\langle A_{i} x_{n_{l}}, x-x_{n_{l}}\right\rangle+\xi_{l} \geq 0, \quad \forall l \geq N_{l}, i=1,2, \ldots, N,
$$

where the existence of $N_{l}$ follows from (i). This means that

$$
\left\langle A_{i} x_{n_{l}}, x+\xi_{l} t_{n_{l}}^{i}-x_{n_{l}}\right\rangle \geq 0, \quad \forall l \geq N_{l}, i=1,2, \ldots, N,
$$

for some $t_{n_{l}}^{i} \in H$ satisfying $1=\left\langle A_{i} x_{n_{l}}, t_{n_{l}}^{i}\right\rangle$ (since $A_{i} x_{n_{l}} \neq 0$ for $i=1,2, \ldots, N$ ). As $A_{i}$ are pseudomonotone, it follows from (i) that

$$
\left\langle A_{i}\left(x+\xi_{l} t_{n_{l}}^{i}\right), x+\xi_{l} t_{n_{l}}^{i}-x_{n_{l}}\right\rangle \geq 0 \quad \forall l \geq N_{l}, i=1,2, \ldots, N .
$$

Furthermore, $x_{n_{l}} \rightarrow p$ as $l \rightarrow \infty$ and $A_{i}$ are weakly sequentially continuous, then $A_{i} x_{n_{l}} \rightarrow A_{i} p$ for $i=1,2, \ldots, N$. Therefore,

$$
0<\left\|A_{i} p\right\| \leq \liminf _{l \rightarrow \infty}\left\|A_{i} x_{n_{l}}\right\|, \quad \forall i=1,2, \ldots, N .
$$

Moreover, $\left\{x_{N_{l}}\right\} \subset\left\{x_{n_{l}}\right\}$ and $\xi_{l} \rightarrow 0$ as $l \rightarrow \infty$. Thus, we obtain

$$
\begin{aligned}
0 & \leq \limsup _{l \rightarrow \infty}\left\|\xi_{l} t_{n_{l}}^{i}\right\|=\limsup _{l \rightarrow \infty}\left(\frac{\xi_{l}}{\left\|A_{i} x_{n_{l}}\right\|}\right) \\
& \leq \frac{\limsup _{l \rightarrow \infty} \xi_{l}}{\liminf _{l \rightarrow \infty}\left\|A_{i} x_{n_{l}}\right\|} \leq \frac{0}{\left\|A_{i} p\right\|}=0
\end{aligned}
$$

which implies that $\lim _{l \rightarrow \infty}\left\|\xi_{l} t_{n_{l}}^{i}\right\|=0$. Thus, taking limit of (20) as $l \rightarrow \infty$, we obtain

$$
\left\langle A_{i} x, x-p\right\rangle \geq 0, \quad \forall i=1,2, \ldots, N .
$$

Using Lemma 1, we have $p \in V I\left(C, A_{i}\right)$ for all $i=1,2, \ldots, N$. Therefore $p \in \bigcap_{i=1}^{N} V I\left(C, A_{i}\right)$. This completes the proof.

We now present our main result.

Theorem 1. Suppose $\left\{x_{n}\right\}$ is generated by Algorithm 3. Then $\left\{x_{n}\right\}$ converges strongly to a point $z \in$ Sol.

Proof. Let $u^{*} \in$ Sol and put $\Gamma_{n}=\left\|x_{n}-x^{*}\right\|^{2}$. We consider the following possible cases.

Case A: Assume that there exists $n_{0} \in \mathbb{N}$ such that $\left\{\Gamma_{n}\right\}$ is monotonically decreasing for $n \geq n_{0}$. Since $\left\{\Gamma_{n}\right\}$ is bounded, then

$$
\Gamma_{n}-\Gamma_{n+1} \rightarrow 0 \text { as } n \rightarrow \infty
$$


Moreover, from Lemma 8 (i), we have

$$
\begin{aligned}
\left\|x_{n+1}-u^{*}\right\|^{2}= & \left\|\alpha_{n} \gamma f\left(x_{n}\right)-\left(1-\alpha_{n} \sum_{n=0}^{\bar{N}} c_{k} B_{k}\right) u_{n}-u^{*}\right\|^{2} \\
= & \left\|\alpha_{n}\left(\gamma f\left(x_{n}\right)-\sum_{n=0}^{\bar{N}} c_{k} B_{k} u^{*}\right)+\left(1-\alpha_{n} \sum_{n=0}^{\bar{N}} c_{k} B_{k}\right)\left(u_{n}-u^{*}\right)\right\|^{2} \\
\leq & \left\|\left(I-\alpha_{n} \sum_{n=0}^{\bar{N}} c_{k} B_{k}\right)\left(u_{n}-u^{*}\right)\right\|^{2}+2 \alpha_{n}\left\langle\gamma f\left(x_{n}\right)-\alpha_{n} \sum_{n=0}^{\bar{N}} c_{k} B_{k} u^{*}, x_{n+1}-u^{*}\right\rangle \\
\leq & \left(1-\alpha_{n} \sum_{n=0}^{\bar{N}} c_{k} \gamma_{k}\right)\left\|u_{n}-u^{*}\right\|^{2}+2 \alpha_{n}\left\langle\gamma f\left(x_{n}\right)-\alpha_{n} \sum_{n=0}^{\bar{N}} c_{k} B_{k} u^{*}, x_{n+1}-u^{*}\right\rangle \\
\leq & \left(1-\alpha_{n} \sum_{n=0}^{\bar{N}} c_{k} \bar{\gamma}\right)\left\|w_{n}-u^{*}\right\|^{2}+2 \alpha_{n}\left\langle\gamma f\left(x_{n}\right)-\alpha_{n} \sum_{n=0}^{\bar{N}} c_{k} B_{k} u^{*}, x_{n+1}-u^{*}\right\rangle \\
\leq & \left(1-\alpha_{n} \bar{\gamma}\right)\left[\left\|x_{n}-u^{*}\right\|^{2}-\frac{\sigma \rho^{l_{n}}}{L} \sum_{i=1}^{N} \beta_{n}^{i}\left\|x_{n}-y_{n}^{i}\right\|^{2}\right] \\
& +2 \alpha_{n}\left\langle\gamma f\left(x_{n}\right)-\alpha_{n} \sum_{n=0}^{\bar{N}} c_{k} B_{k} u^{*}, x_{n+1}-u^{*}\right\rangle .
\end{aligned}
$$

Therefore

$$
\begin{aligned}
\left(1-\alpha_{n} \bar{\gamma}\right) \frac{\sigma \rho^{l_{n}}}{L} \sum_{i=1}^{N} \beta_{n}^{i}\left\|x_{n}-y_{n}^{i}\right\|^{2} \leq & \left(1-\alpha_{n} \bar{\gamma}\right)\left\|x_{n}-u^{*}\right\|^{2}-\left\|x_{n+1}-u^{*}\right\|^{2} \\
& +2 \alpha_{n}\left\langle\gamma f\left(x_{n}\right)-\alpha_{n} \sum_{n=0}^{\bar{N}} c_{k} B_{k} u^{*}, x_{n+1}-u^{*}\right\rangle .
\end{aligned}
$$

As $\alpha_{n} \rightarrow 0$ as $n \rightarrow \infty$ and from (21), we have

$$
\lim _{n \rightarrow \infty} \frac{\sigma \rho^{l_{n}}}{L} \sum_{i=1}^{N} \beta_{n}^{i}\left\|x_{n}-y_{n}^{i}\right\|^{2}=0 .
$$

Furthermore, from (18), we obtain

$$
\begin{aligned}
\left\|x_{n+1}-u^{*}\right\|^{2} \leq & \left(1-\alpha_{n} \bar{\gamma}\right)\left\|u_{n}-u^{*}\right\|^{2}+2 \alpha_{n}\left\langle\gamma f\left(x_{n}\right)-\alpha_{n} \sum_{n=0}^{\bar{N}} c_{k} B_{k} u^{*}, x_{n+1}-u^{*}\right\rangle \\
\leq & \left(1-\alpha_{n} \bar{\gamma}\right)\left[\left\|x_{n}-u^{*}\right\|^{2}-\sum_{j=1}^{M}\left(\delta_{n, 0}-\kappa\right) \delta_{n, j}\left\|w_{n}-T_{j} w_{n}\right\|^{2}\right] \\
& +2 \alpha_{n}\left\langle\gamma f\left(x_{n}\right)-\alpha_{n} \sum_{n=0}^{\bar{N}} c_{k} B_{k} u^{*}, x_{n+1}-u^{*}\right\rangle .
\end{aligned}
$$

This implies that

$$
\begin{aligned}
\left(1-\alpha_{n} \bar{\gamma}\right) \sum_{j=1}^{M}\left(\delta_{n, 0}-\kappa\right) \delta_{n, j}\left\|w_{n}-T_{j} w_{n}\right\|^{2} \leq & \left(1-\alpha_{n} \bar{\gamma}\right)\left\|x_{n}-u^{*}\right\|^{2}-\left\|x_{n+1}-u^{*}\right\|^{2} \\
& +2 \alpha_{n}\left\langle\gamma f\left(x_{n}\right)-\alpha_{n} \sum_{n=0}^{N} c_{k} B_{k} u^{*}, x_{n+1}-u^{*}\right\rangle .
\end{aligned}
$$


Therefore,

$$
\lim _{n \rightarrow \infty} \sum_{j=1}^{M}\left(\delta_{n, 0}-\kappa_{j}\right) \delta_{n, j}\left\|w_{n}-T_{j} w_{n}\right\|=0 .
$$

Using condition (C2), we obtain

$$
\lim _{n \rightarrow \infty}\left\|w_{n}-T_{j} w_{n}\right\|=0
$$

Consequently,

$$
\left\|u_{n}-w_{n}\right\| \leq \delta_{n, 0}\left\|w_{n}-w_{n}\right\|+\sum_{j=1}^{M} \delta_{n . j}\left\|w_{n}-T_{j} w_{n}\right\| \rightarrow 0 \quad \text { as } n \rightarrow \infty .
$$

Furthermore, from (11), we have

$$
\begin{aligned}
\left\|w_{n}-u^{*}\right\|^{2} & =\left\|P_{D_{n}}\left(x_{n}\right)-u^{*}\right\|^{2} \\
& \leq\left\|x_{n}-u^{*}\right\|^{2}-\left\|w_{n}-x_{n}\right\|^{2} .
\end{aligned}
$$

Then, from (23), we have

$$
\begin{aligned}
\left\|w_{n}-x_{n}\right\|^{2} \leq & \left\|x_{n}-u^{*}\right\|^{2}-\left\|w_{n}-u^{*}\right\|^{2} \\
= & \left\|x_{n}-u^{*}\right\|^{2}-\left\|x_{n+1}-u^{*}\right\|^{2}+\left\|x_{n+1}-u^{*}\right\|^{2}-\left\|w_{n}-u^{*}\right\|^{2} \\
\leq & \left\|x_{n}-u^{*}\right\|^{2}-\left\|x_{n+1}-u^{*}\right\|^{2}+\left\|w_{n}-u^{*}\right\|^{2}+2 \alpha_{n}\left\langle\gamma f\left(x_{n}\right)-\alpha_{n} \sum_{n=0}^{\bar{N}} c_{k} B_{k} u^{*}, x_{n+1}-u^{*}\right\rangle \\
& \quad-\left\|w_{n}-u^{*}\right\|^{2} \\
= & \left\|x_{n}-u^{*}\right\|^{2}-\left\|x_{n+1}-u^{*}\right\|^{2}+2 \alpha_{n}\left\langle\gamma f\left(x_{n}\right)-\alpha_{n} \sum_{n=0}^{\bar{N}} c_{k} B_{k} u^{*}, x_{n+1}-u^{*}\right\rangle .
\end{aligned}
$$

Moreover, as $\alpha_{n} \rightarrow 0$ as $n \rightarrow \infty$, then

$$
\lim _{n \rightarrow \infty}\left\|w_{n}-x_{n}\right\|=0
$$

From (25) and (26), we get

$$
\left\|u_{n}-x_{n}\right\| \leq\left\|u_{n}-w_{n}\right\|+\left\|w_{n}-x_{n}\right\| \rightarrow 0 \text { as } n \rightarrow \infty .
$$

Therefore,

$$
\begin{aligned}
\left\|x_{n+1}-x_{n}\right\| & =\left\|\alpha_{n}\left(\gamma f\left(x_{n}\right)-\sum_{n=0}^{\bar{N}} c_{k} B_{k} x_{n}\right)+\left(I-\alpha_{n} \sum_{n=0}^{\bar{N}}\right)\left(u_{n}-x_{n}\right)\right\| \\
& \leq \alpha_{n}\left\|\gamma f\left(x_{n}\right)-\sum_{n=0}^{\bar{N}} c_{k} B_{k} x_{n}\right\|+\left(1-\alpha_{n} \bar{\gamma}\right)\left\|u_{n}-x_{n}\right\| \rightarrow 0 \quad \text { as } n \rightarrow \infty .
\end{aligned}
$$

Now, we show that $\Omega_{w}\left(x_{n}\right) \subset$ Sol, where $\Omega_{w}\left(x_{n}\right)$ is the set of weak subsequential limits of $\left\{x_{n}\right\}$. Let $p \in \Omega_{w}\left(x_{n}\right)$, then there exists a subsequence $\left\{x_{n_{l}}\right\}$ of $\left\{x_{n}\right\}$ such that $x_{n_{l}} \rightarrow p$ as $l \rightarrow \infty$. Let $\left\{y_{n_{l}}^{i}\right\}$ be subsequences of $\left\{y_{n}^{i}\right\}$ for $i=1,2, \ldots, N$. From (23), we have

$$
\lim _{l \rightarrow \infty} \gamma^{m_{n_{l}}} \sum_{i=1}^{N} \beta_{n_{l}}\left\|x_{n_{l}}-y_{n_{l}}^{i}\right\|=0, \quad \forall i=1,2, \ldots, N .
$$


Now we claim that

$$
\lim _{l \rightarrow \infty}\left\|x_{n_{l}}-y_{n_{l}}^{i}\right\|=0, \quad \forall i=1,2, \ldots, N
$$

Indeed, we consider two distinct cases depending on the behavior of subsequence $\left\{\gamma^{m_{n_{l}}}\right\}$. (i) If $\liminf _{l \rightarrow \infty} \gamma^{m_{n_{l}}}>0$, then

$$
0 \leq\left\|x_{n_{l}}-y_{n_{l}}^{i}\right\|^{2}=\frac{\gamma^{m_{n_{l}}}\left\|x_{n_{l}}-y_{n_{l}}\right\|^{2}}{\gamma^{m_{n_{l}}}}
$$

This implies that

$$
\begin{aligned}
\limsup _{l \rightarrow \infty}\left\|x_{n_{l}}-y_{n_{l}}\right\|^{2} & \leq \limsup _{l \rightarrow \infty}\left(\gamma^{m_{n_{l}}}\left\|x_{n_{l}}-y_{n_{l}}\right\|^{2}\right)\left(\limsup _{l \rightarrow \infty} \frac{1}{\gamma^{m_{n_{l}}}}\right) \\
& =\limsup _{l \rightarrow \infty}\left(\gamma^{m_{n_{l}}}\left\|x_{n_{l}}-y_{n_{l}}\right\|^{2}\right)\left(\frac{1}{\liminf _{l \rightarrow \infty} \gamma^{m_{n_{l}}}}\right) \\
& =0 .
\end{aligned}
$$

Therefore,

$$
\lim _{l \rightarrow \infty}\left\|x_{n_{l}}-y_{n_{l}}^{i}\right\|=0
$$

(ii) Suppose $\lim \inf _{l \rightarrow \infty} \gamma^{m_{n_{l}}}=0$. Then we may assume without loss of generality that $\lim _{l \rightarrow \infty} \gamma^{m_{n_{l}}}=0$ and $\lim _{l \rightarrow \infty}\left\|x_{n_{l}}-y_{n_{l}}\right\|=a>0$. Let us define $\bar{z}_{n_{l}}^{i}=\frac{1}{t} \gamma^{m_{n_{l}}} y_{n_{l}}^{i}+\left(1-\frac{1}{t} \gamma^{m_{n_{l}}}\right) x_{n}$ for $i=1,2, \ldots, N$. This implies that $\bar{z}_{n_{l}}^{i}-x_{n}=\frac{1}{t} \gamma^{m_{n_{l}}}\left(y_{n_{l}}^{i}-x_{n_{l}}\right)$ for $i=1,2, \ldots, N$. Since $\left\{y_{n_{l}}^{i}-x_{n_{l}}\right\}$ are bounded and $\lim _{l \rightarrow \infty} \gamma^{m_{n_{l}}}=0$, then

$$
\lim _{l \rightarrow \infty}\left\|\bar{z}_{n_{l}}^{i}-x_{n_{l}}\right\|=0 .
$$

As $A_{i}$ are uniformly continuous, then

$$
\lim _{l \rightarrow \infty}\left\|A_{i} \bar{z}_{n_{l}}^{i}-A_{i} x_{n_{l}}\right\|=0, \quad \forall i=1,2, \ldots, N .
$$

Using (12) and from the Definition of $\bar{z}_{n_{l}}^{i}$ for $i=1,2, \ldots, N$, we know that

$$
\left\langle A_{i} \bar{z}_{n_{l}}^{i}, x_{n_{l}}-y_{n_{l}}^{i}\right\rangle<\frac{\sigma}{2}\left\|x_{n_{l}}-y_{n_{l}}^{i}\right\|^{\prime} \quad \forall i=1,2, \ldots, N
$$

Therefore,

$$
2\left\langle A_{i} x_{n_{l}}, x_{n_{l}}-y_{n_{l}}^{i}\right\rangle+2\left\langle A_{i} \bar{z}_{n_{l}}^{i}-A_{i} x_{n_{l}}, x_{n_{l}}-y_{n_{l}}^{i}\right\rangle<\sigma\left\|x_{n_{l}}-y_{n_{l}}^{i}\right\|^{2}, \quad \forall i=1,2, \ldots, N
$$

Putting $v_{n_{l}}^{i}=x_{n_{l}}-A_{i} x_{n_{l}}$, for all $i=1,2, \ldots, N$, we obtain

$$
2\left\langle x_{n_{l}}-v_{n_{l}}^{i}, x_{n_{l}}-y_{n_{l}}^{i}\right\rangle+2\left\langle A_{i} z_{n_{l}}^{i}-A_{i} x_{n_{l}}, x_{n_{l}}-y_{n_{l}}^{i}\right\rangle<\sigma\left\|x_{n_{l}}-y_{n_{l}}^{i}\right\|^{2}, \quad \forall i=1,2, \ldots, N .
$$

Moreover, from Lemma 2 (i), we have

$$
2\left\langle x_{n_{l}}-y_{n_{l}}, x_{n_{l}}-y_{n_{l}}^{i}\right\rangle=\left\|x_{n_{l}}-v_{n_{l}}^{i}\right\|^{2}+\left\|x_{n_{l}}-y_{n_{l}}^{i}\right\|^{2}-\left\|y_{n_{l}}^{i}-v_{n_{l}}^{i}\right\|^{2} .
$$

Substituting (30) into (29), we have

$$
\left\|x_{n_{l}}-v_{n_{l}}^{i}\right\|^{2}-\left\|y_{n_{l}}^{i}-v_{n_{l}}^{i}\right\|^{2}<(\sigma-1)\left\|x_{n_{l}}-y_{n_{l}}^{i}\right\|^{2}-2\left\langle A_{i} \bar{z}_{n_{l}}^{i}-A_{i} x_{n_{l}}, x_{n_{l}}-y_{n_{l}}^{i}\right\rangle .
$$


Passing limit to the last inequality as $l \rightarrow \infty$ and using (28), we get

$$
\lim _{l \rightarrow \infty}\left(\left\|x_{n_{l}}-v_{n_{l}}^{i}\right\|^{2}-\left\|y_{n_{l}}^{i}-v_{n_{l}}^{i}\right\|^{2}\right) \leq(\sigma-1) a<0
$$

For $\epsilon=\frac{-(\sigma-1) a}{2}>0$, there exists $m \in \mathbb{N}$ such that

$$
\left\|x_{n_{l}}-v_{n_{l}}^{i}\right\|^{2}-\left\|y_{n_{l}}^{i}-v_{n_{l}}^{i}\right\|^{2} \leq(\sigma-1) a+\epsilon=\frac{(\sigma-1) a}{2}<0
$$

for all $l \in \mathbb{N}, n \geq m, i=1,2, \ldots, N$. Therefore

$$
\left\|x_{n_{l}}-v_{n_{l}}^{i}\right\|^{2}<\left\|y_{n_{l}}^{i}-v_{n_{l}}^{i}\right\|^{2}, \quad \forall l \in \mathbb{N}, n \geq m, i=1,2, \ldots, N .
$$

This contradicts the Definition of metric projection as $y_{n_{l}}^{i}=P_{C}\left(x_{n_{l}}-A_{i} x_{n_{l}}\right)$. Thus $a=0$. Therefore, we obtain

$$
\lim _{l \rightarrow \infty}\left\|x_{n_{l}}-y_{n_{l}}^{i}\right\|=0, \quad \forall i=1,2, \ldots, N
$$

Consequently from Lemma 9, we have $p \in \bigcap_{i=1}^{N} V I\left(C, A_{i}\right)$. Furthermore, as $w_{n, l} \rightarrow p$ and $\left\|v_{n_{l}, j}-w_{n_{l}}\right\| \rightarrow 0$, then by the demi-closedness of $T_{j}, j=1,2, \ldots, M$, we have that $p \in \operatorname{Fix}\left(T_{j}\right)$, for each $j=1,2, \ldots, M$. This means that $p \in \bigcap_{j=1}^{M} \operatorname{Fix}\left(T_{j}\right)$. Therefore, $p \in$ Sol, which show that $\Omega_{w}\left(x_{n}\right) \subset$ Sol. We now show that $\left\{x_{n}\right\}$ converges strongly to a point $u^{*} \in$ Sol. As $x_{n_{l}} \rightarrow p$ and $\left\|x_{n_{l}+1}-x_{n_{l}}\right\| \rightarrow 0$ as $l \rightarrow \infty$, then $x_{n_{l}+1} \rightarrow p$. Therefore,

$$
\begin{aligned}
\limsup _{n \rightarrow \infty}\left\langle\gamma f\left(u^{*}\right)-\sum_{n=0}^{\bar{N}} c_{k} B_{k} u^{*}, x_{n+1}-u^{*}\right\rangle & =\lim _{l \rightarrow \infty}\left\langle\gamma f\left(u^{*}\right)-\sum_{n=0}^{\bar{N}} c_{k} B_{k} u^{*}, x_{n_{l}+1}-u^{*}\right\rangle \\
& =\left\langle\gamma f\left(u^{*}\right)-\sum_{n=0}^{\bar{N}} c_{k} B_{k} u^{*}, p-u^{*}\right\rangle .
\end{aligned}
$$

As $p \in$ Sol, then it follows from (10) and (32) that

$$
\limsup _{n \rightarrow \infty}\left\langle\gamma f\left(u^{*}\right)-\sum_{n=0}^{\bar{N}} c_{k} B_{k} u^{*}, x_{n+1}-u^{*}\right\rangle \leq 0
$$

Therefore, using Lemma 4 and Lemma 8 (ii), we have that $\lim _{n \rightarrow \infty}\left\|x_{n}-u^{*}\right\|=0$. This implies that $\left\{x_{n}\right\}$ converges strongly to $u^{*}$.

Case B: Suppose $\left\{\Gamma_{n}\right\}$ is not monotonically decreasing. Let $\tau: \mathbb{N} \rightarrow \mathbb{N}$ for all $n \geq n_{0}$ (for some $n_{0}$ large enough) be defined by

$$
\tau(n)=\max \left\{k \in \mathbb{N}: k \leq n, \Gamma_{k} \leq \Gamma_{k+1}\right\} .
$$

Clearly $\tau$ is non-decreasing, $\tau(n) \rightarrow \infty$ as $n \rightarrow \infty$ and

$$
0 \leq \Gamma_{\tau(n)} \leq \Gamma_{\tau(n)+1}, \quad \forall n \geq n_{0}
$$

As $\left\{x_{\tau(n)}\right\}$ is bounded, there exists a subsequence of $\left\{x_{\tau(n)}\right\}$ still denoted by $\left\{x_{\tau(n)}\right\}$ which converges weakly to $p \in C$. Following similar arguments as in Case $\mathrm{A}$, we get

$$
\begin{array}{r}
\lim _{n \rightarrow \infty}\left\|w_{\tau(n)}-x_{\tau(n)}\right\|=\lim _{n \rightarrow \infty}\left\|u_{\tau(n)}-x_{\tau(n)}\right\|=\lim _{n \rightarrow \infty}\left\|x_{\tau(n)+1}-x_{\tau(n)}\right\|=0, \\
\lim _{n \rightarrow \infty}\left\|x_{\tau(n)}-y_{\tau(n)}^{i}\right\|=0, \quad \forall i=1,2 \ldots, N, \lim _{n \rightarrow \infty}\left\|v_{\tau(n), j}-w_{\tau(n)}\right\|=0, \quad j=1,2, \ldots, M,
\end{array}
$$


and $\Omega_{w}\left(x_{\tau(n)}\right) \subset$ Sol, where $\Omega_{w}\left(x_{\tau(n)}\right)$ is the set of weak subsequential limits of $\left\{x_{\tau(n)}\right\}$. Furthermore,

$$
\limsup _{n \rightarrow \infty}\left\langle\gamma f\left(u^{*}\right)-\sum_{n=0}^{\bar{N}} c_{k} B_{k} u^{*}, x_{\tau(n)+1}-u^{*}\right\rangle \leq 0
$$

From Lemma 8 (ii), we have

$$
\begin{aligned}
\left\|x_{\tau(n)+1}-u^{*}\right\|^{2} \leq & \left(1-\frac{2 \alpha_{\tau(n)}(\bar{\gamma}-\alpha \gamma)}{1-\alpha_{\tau(n)} \alpha \gamma}\right)\left\|x_{\tau(n)}-u^{*}\right\|^{2} \\
& +\frac{2 \alpha_{n}(\bar{\gamma}-\alpha \gamma)}{1-\alpha_{\tau(n)} \alpha \gamma}\left(\alpha_{\tau(n)} M+\frac{\left\langle\gamma f\left(u^{*}\right)-\sum_{k=0}^{\bar{N}} c_{k} B_{k} u^{*}, x_{\tau(n)+1}-u^{*}\right\rangle}{\bar{\gamma}-\alpha_{\tau(n)} \gamma}\right),
\end{aligned}
$$

for some $M>0$. As $0 \leq\left\|x_{\tau(n)}-u^{*}\right\|^{2} \leq\left\|x_{\tau(n)+1}-u^{*}\right\|^{2}$, then we get

$$
\left\|x_{\tau(n)}-u^{*}\right\|^{2} \leq \alpha_{\tau(n)} M+\frac{\left\langle\gamma f\left(u^{*}\right)-\sum_{k=0}^{\bar{N}} c_{k} B_{k} u^{*}, x_{\tau(n)+1}-u^{*}\right\rangle}{\bar{\gamma}-\alpha_{\tau(n)} \gamma} .
$$

Then from (33) and the fact that $\alpha_{\tau(n)} \rightarrow 0$, we have

$$
\lim _{n \rightarrow \infty}\left\|x_{\tau(n)}-u^{*}\right\|=0 .
$$

Furthermore, for $n \geq n_{0}$, it is easy to see that $\Gamma_{n} \leq \Gamma_{\tau(n)+1}$. As a consequence, we get for all sufficiently large $n$ that $0 \leq \Gamma_{n} \leq \Gamma_{\tau(n)+1}$. Thus, $\lim _{n \rightarrow \infty}\left\|x_{n}-u^{*}\right\|=0$. Therefore, $\left\{x_{n}\right\}$ converges strongly to $u^{*}$. Consequently, $\left\{y_{n}^{i}\right\},\left\{z_{n}^{i}\right\},\left\{w_{n}\right\}$ and $\left\{u_{n}\right\}$ converges strongly to $u^{*}$. This completes the proof.

\section{Remark 3.}

(i) Instead of finding the farthest element to the iterate $x_{n}$, we construct a sub-level set using the convex combination of the finite functions and perform a single projection onto the sub-level set. Note that this projection can be calculated explicitly irrespective of the feasible set $C$.

(ii) We emphasize that the convergence of our Algorithm 3 is proved without using a prior estimate of any Lipshitz constant. Moreover, the cost operators do not even need to satisfy the Lipschitz condition. Note that the previous results of $[6,8,33]$ and references therein cannot be applied in this situation.

(iii) We give an example of a finite family of $A_{i}: H \rightarrow H$ which does not satisfy Lipschitz condition.

Example 1. Let $H=\Re^{n}$ defined by $\Re^{n}=\left\{\bar{x}=\left(x_{1}, x_{2}, \ldots, x_{n}\right), x_{l} \in \Re: \sum_{l=1}^{n}\left|x_{l}\right|^{2}<\infty\right\}$ with norm $\|\cdot\|: \Re^{n} \rightarrow[0 . \infty)$ defined by $\|\bar{x}\|:=\left(\sum_{l=1}^{n}\left|x_{l}\right|^{2}\right)^{\frac{1}{2}}$ for arbitrary $\bar{x}=\left(x_{1}, x_{2}, \ldots, x_{n}\right) \in \Re^{n}$. Let $C_{i}=C=\left\{\bar{x} \in \Re^{n}:\|\bar{x}\| \leq 1\right\}$ and for $i=1, \ldots, N$, let $A_{i}: C \rightarrow H$ be defined by

$$
A_{i} x=\left(\|x\|+\frac{i}{\|x\|+1}\right) x, \quad i=1, \ldots, N .
$$

It is clear that $\operatorname{VI}\left(C, A_{i}\right) \neq \varnothing$ as $0 \in \operatorname{VI}\left(C, A_{i}\right)$ for each $i=1, \ldots, N$. First, we show that $A_{i}$ is pseudomonotone and not Lipschitz continuous for $i=1,2, \ldots, N$. Let $u, v \in C$ be such that $\left\langle A_{i} u, v-u\right\rangle \geq 0$. This means that $\langle u, v-u\rangle \geq 0$. Thus, 


$$
\begin{aligned}
\left\langle A_{i} v, v-u\right\rangle & =\left(\|v\|+\frac{i}{\|v\|+1}\right)\langle v, v-u\rangle \\
& >\left(\|v\|+\frac{i}{\|v\|+1}\right)(\langle v, v-u\rangle-\langle u, v-u\rangle) \\
& =\left(\|v\|+\frac{i}{\|v\|+1}\right)\|v-u\|^{2}>0 .
\end{aligned}
$$

Therefore, $A_{i}$ is pseudomonotone for $i=1, \ldots, N$. To see that $A_{i}$ is not L-Lipschitz continuous for $i=1,2, \ldots, N$, let $u=\left(L_{i}, 0, \ldots, 0\right)$ and $v=(0,0, \ldots, 0)$. Then,

$$
\|A u-A v\|=\|A u\|=\left(\|u\|+\frac{i}{\|u\|+1}\right)\|u\|=\left(L_{i}+\frac{i}{L_{i}+1}\right) L_{i} .
$$

Moreover, $\left\|A_{i} u-A_{i} v\right\| \leq L_{i}\|u-v\|$ implies that

$$
\left(L_{i}+\frac{i}{L_{i}+1}\right) L_{i} \leq L_{i}^{2}
$$

Thus, $\frac{i}{L_{i}+1} \leq 0$, which is a contradiction. Therefore, $A_{i}$ is not Lipschitz continuous for $i=1, \ldots, N$.

\section{Numerical Experiments}

In this section, we present some numerical experiments to illustrate the performance of the proposed algorithm. We compare our Algorithm 3 with Algorithm 1 of Anh and Phuong [8], Algorithm 2 of Hieu [33], Algorithm 1 of Suantai et al. [40], and other algorithms in the literature. The projections onto $C_{i}$ are computed explicitly. All codes are written with a HP PC with the following specification: Intel(R)core i7-9700, CPU 3.00GHz, RAM 4.0GB, MATLAB version 9.9 (R2020b).

Example 2. First, we consider the variational inequalities with operators $A_{i}: \Re^{m} \rightarrow \Re^{m}$ for $i=1,2, \ldots, N$, defined by $A_{i}(x)=G_{i}(x)+q_{i}$, where

$$
G_{i}=S_{i} S_{i}^{T}+Q_{i}+R_{i}, \quad i=1,2, \ldots, N,
$$

such that for each $i, S_{i}$ is a $m \times m$ matrix, $Q_{i}$ is a $m \times m$ skew symmetric matrix, $R_{i}$ is a $m \times m$ diagonal matrix, whose diagonal entries are non-negative (so $G_{i}$ is positive definite) and $q_{i}$ is a vector in $\Re^{m}$. The feasible set $C$ is given by $C_{i}=C=\left\{x \in \Re^{m}:\langle x, a\rangle \leq c\right\}$, where $a \in \Re^{m}$ is generated randomly and $c$ is a positive real number randomly in $[1, m]$. It is clear that for each $i, G_{i}$ is monotone (hence, pseudomonotone) and Lipschitz continuous with Lipschitz constant $L_{i}=\max \left\{\left\|G_{i}\right\|: i=1,2, \ldots, N\right\}$. The entries of matrices $S_{i}, Q_{i}, R_{i}$ are generated randomly and uniformly in $[-m, m]$, diagonal entries of $R_{i}$ are in $[1, m]$ and $q_{i}$ is equal to the zero vector. In this case, it is easy to see that the $\operatorname{VI}\left(C, A_{i}\right)=\{0\}$. For $j=1,2, \ldots, M$, let $T_{j}: \Re^{m} \rightarrow \Re^{m}$ be defined by $T_{j} x=\frac{x}{2 j}$, for $j \in \mathbb{N}$. Then $T_{j}$ is 0-demicontractive mapping, $\operatorname{Fix}\left(T_{j}\right)=\{0\}$ and $\left(I-T_{j}\right)$ is demiclosed at 0 . Also for $k=1,2, \ldots, \bar{N}$, let $B_{k}=\frac{1}{2 k} I, f=I$ ( $I$ being the identity operator on $H$ ), we choose $\alpha=1, \gamma=\frac{1}{8}, \sigma=0.28, \rho=0.36, \lambda=\frac{1}{4}, c_{k}=\frac{1}{\bar{N}}, \delta_{n, j}=\frac{1}{M+1}, \alpha_{n}=\frac{1}{n+1}, \beta_{n, i}=\frac{1}{N+1}$, for all $n \in \mathbb{N}$. We compare Algorithm 3 with Algorithm 1 of Anh and Phuong [8], Algorithm 2 of Hieu [33], and Algorithm 1 of Suantai et al. [40]. We test the algorithms using the following parameters.

- Anh and Phuong alg.: $\lambda_{n, i}=\frac{0.99}{2 L_{i}}, \alpha_{n, i}=\frac{1}{n i+1}, \gamma_{n, i}=\frac{1}{3}$,

- Hieu alg.: $\lambda=\frac{1}{1.5 L}$,

- Suantai et al. alg.: $\rho=0.34, \mu=0.06$,

and

Case I: $\quad m=5, \quad N=5, \quad M=2, \quad \bar{N}=1$,

Case II: $m=10, \quad N=10, \quad M=5, \quad \bar{N}=5$,

Case III: $m=20, \quad N=15, \quad M=10, \quad \bar{N}=10$, 
Case IV: $m=50, \quad N=20, \quad M=5, \quad \bar{N}=15$.

We also use $\left\|x_{n}-x^{*}\right\|<10^{-5}$ as stopping criterion for each algorithm and plot the graphs of $D_{n}=$ $\left\|x_{n}-x^{*}\right\|^{2}$ against the number of iteration. The computational results are shown in Table 1 and Figure 1.

Table 1. Computational result for Example 2.

\begin{tabular}{llllll}
\hline & & Algorithm 3 & Anh-Phuong [8] & Hieu [33] & Suantai et al. [40] \\
\hline \multirow{2}{*}{ Case I } & No of Iter. & 16 & 34 & 39 & 67 \\
& Time (sec) & 0.0038 & 0.0034 & 0.0032 & 0.0061 \\
\hline \multirow{2}{*}{ Case II } & No of Iter. & 15 & 63 & 107 & 98 \\
& Time (sec) & 0.0020 & 0.0054 & 0.0100 & 0.0097 \\
\hline \multirow{2}{*}{ Case III } & No of Iter. & 14 & 57 & 93 & 183 \\
& Time (sec) & 0.0020 & 0.0053 & 0.0093 & 0.0236 \\
\hline \multirow{2}{*}{ Case IV } & No of Iter. & 10 & 53 & 114 & 183 \\
& Time (sec) & 0.0019 & 0.0047 & 0.0136 & 0.0244 \\
\hline
\end{tabular}
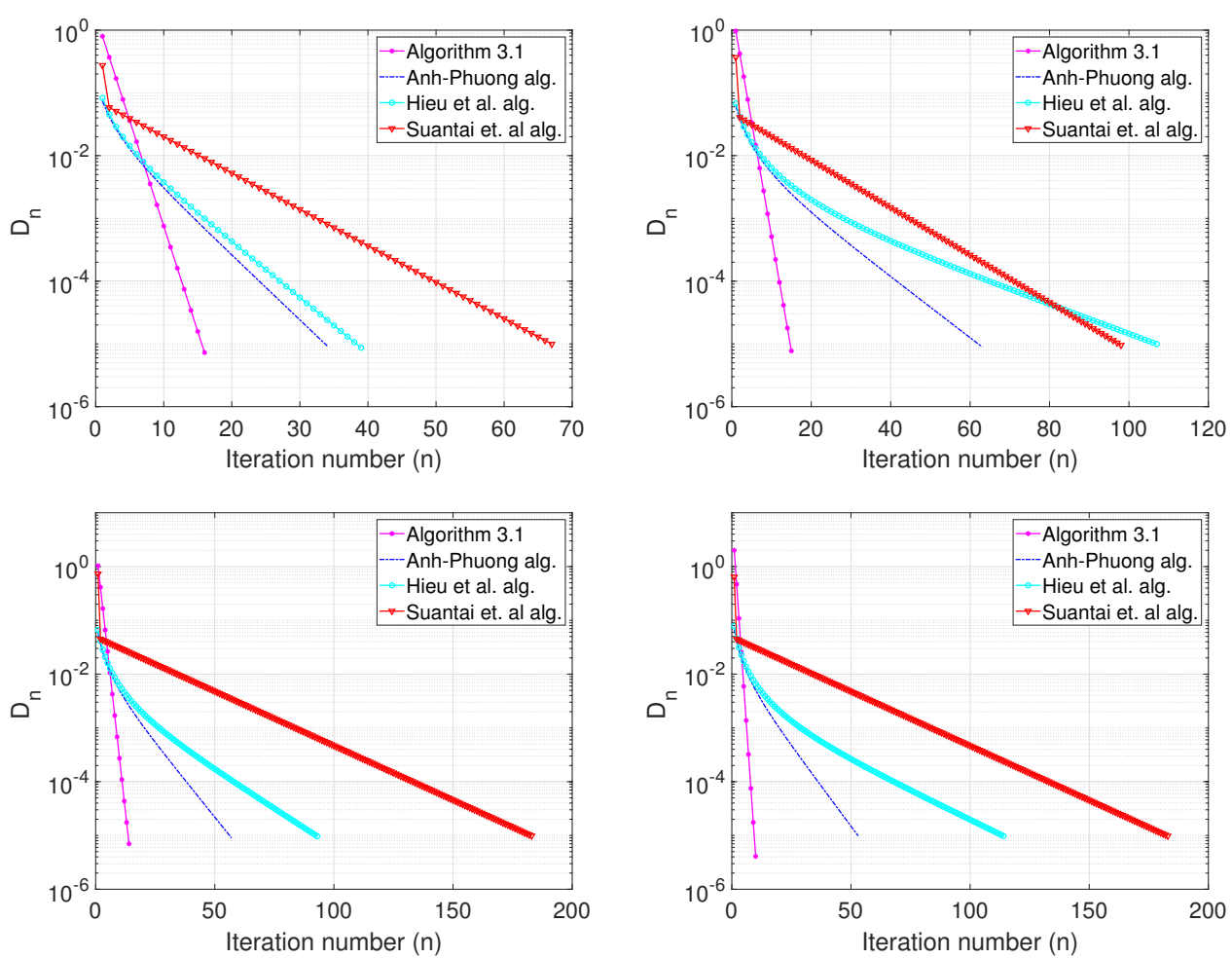

Figure 1. Example 2, From Top to Bottom: Case I, Case II, Case III, and Case IV.

Now, we consider the case when $N=1$ with finite family of demicontractive mappings in infinite dimensional spaces. In this example, we compare our algorithm with Algorithm 1 of Anh et al. [41] and Algorithm 2.1 of Hieu [42].

Example 3. Let $H=\ell_{2}(\Re)$ and define $A: H \rightarrow H$ by $A x=\frac{2}{2+\|x\|}$. It is easy to see that $A$ is easy to see that $A$ is strongly pseudomonotone and Lipschitz continuous with $L=\frac{1}{2}$. We defined the feasible set $C=\left\{x=\left(x_{1}, x_{2}, \ldots\right) \in \ell_{2}:\|x\| \leq 1\right\}$ and for $j=1,2, \ldots, M, T_{j}: H \rightarrow H$ is defined by $T_{j} x=\frac{-(1+j)}{j} x$, for $j \in \mathbb{N}$. Then $T_{j}$ is demicontractive mapping with $\kappa_{j}=\frac{1}{1+2 j}$, Fix $\left(T_{j}\right)=\{0\}$ and $\left(I-T_{j}\right)$ is demiclosed at 0. We choose $\bar{N}=1, B_{k}=\frac{1}{2} I, f(x)=\frac{x}{4}, \sigma=0.02, \rho=0.036, \gamma=\frac{1}{16}, \alpha_{n}=\frac{1}{\sqrt{(n+1)}}, \delta_{n, j}=\frac{1}{M+1}, \beta_{n}^{i}=1$, $c_{k}=1$. For Anh et al. alg., we take $\lambda_{n}=\frac{1}{\sqrt{n+1}}, \alpha_{n}=\frac{1}{2 n+4}$; and for Hieu alg., we take $\lambda_{n}=\frac{1}{n+1}, \beta_{n}^{i}=$ $\frac{n}{2 n+i}, \gamma_{n}^{i}=\frac{1}{M+1}$ (where $i=j$ in this context). We test the algorithms for $M=5,15,20,30$ and study the 
behavior of the sequence generated by the algorithms using $D_{n}=\left\|x_{n}-x^{*}\right\|<10^{-5}$ as stopping criterion. The numerical results are shown in Table 2 and Figure 2.

Table 2. Computational result for Example 2.

\begin{tabular}{lllll}
\hline & & Algorithm 3 & Anh et al. [41] & Hieu [42] \\
\hline \multirow{2}{*}{ N 5} & No of Iter. & 7 & 14 & 67 \\
& Time (sec) & $5.836 \mathrm{e}-04$ & 0.0014 & 0.0021 \\
\hline \multirow{2}{*}{$M=15$} & No of Iter. & 8 & 13 & 25 \\
& Time (sec) & $6.300 \mathrm{e}-04$ & 0.0014 & 0.0018 \\
\hline \multirow{2}{*}{$M=20$} & No of Iter. & 11 & 16 & 37 \\
& Time (sec) & 0.0010 & 0.0033 & 0.0088 \\
\hline \multirow{2}{*}{$M=30$} & No of Iter. & 10 & 17 & 43 \\
& Time (sec) & $7.583 \mathrm{e}-04$ & 0.0018 & 0.0043 \\
\hline
\end{tabular}
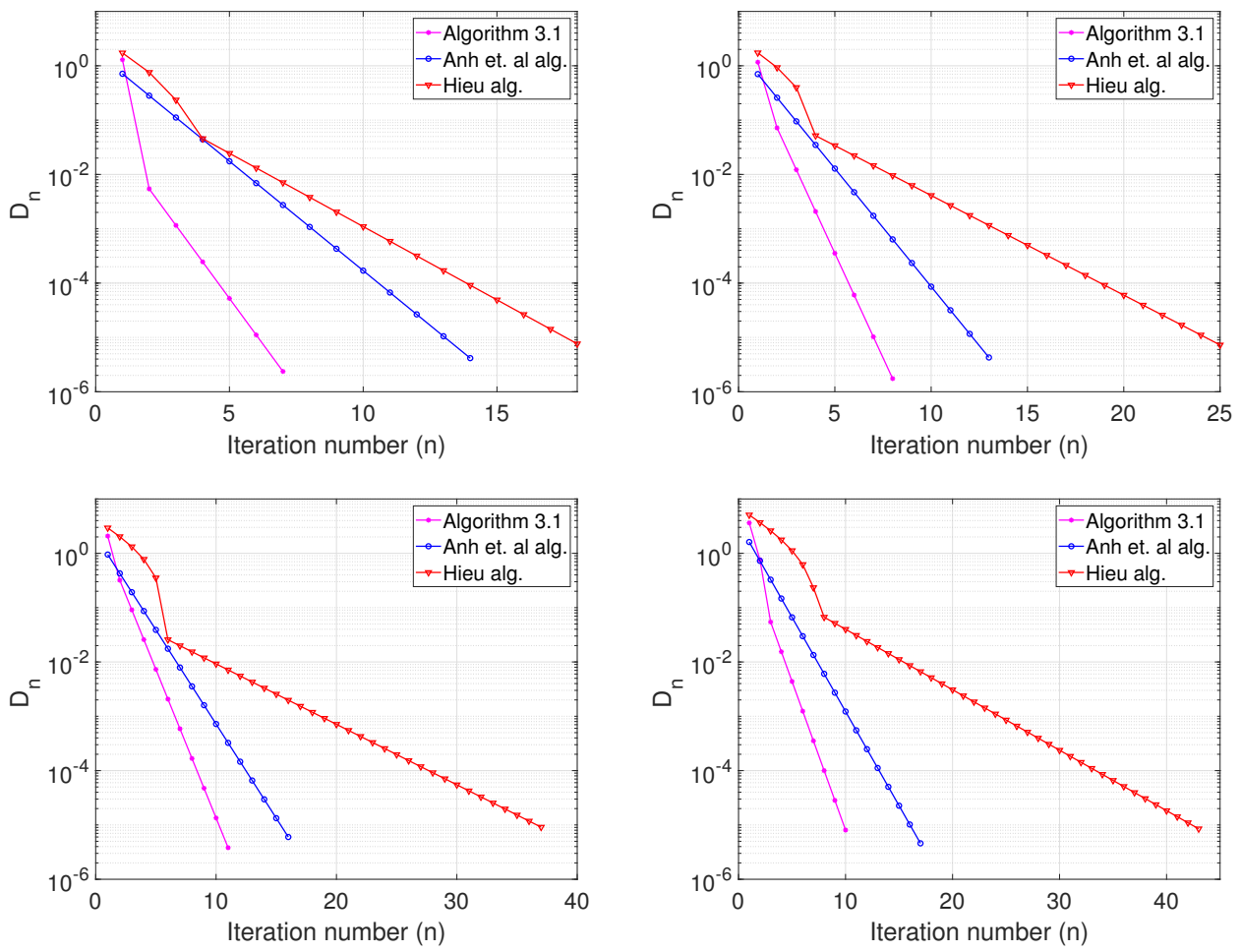

Figure 2. Example 3, From Top to Bottom: $M=5,15,20,30$.

\section{Conclusions}

In this paper, we introduced a new efficient parallel extragradient method for solving systems of variational inequalities involving common fixed point of demicontractive mappings in real Hilbert spaces. The algorithm is designed such that its step size is determined by an Armijo line search technique and a projection onto a sub-level set is computed for determining the next iterate. A strong convergence result is proved under suitable conditions on the control parameters. Finally, some numerical results were reported to show the performance of the proposed method with respect to some other methods in the literature.

Author Contributions: Conceptualization, L.O.J.; Formal analysis, L.O.J.; Funding acquisition, M.A.; Investigation, L.O.J.; Methodology, L.O.J.; Project administration, L.O.J., M.A.; Resources, M.A.; Software, L.O.J.; Supervision, M.A.; Validation, L.O.J.; Visualization, L.O.J.; Writing—original draft, L.O.J.; Writing—review editing, M.A. All authors have read and agreed to the published version of the manuscript. 
Funding: L.O. Jolaoso is supported by the Postdoctoral research grant from the Sefako Makgatho Health Sciences University, South Africa.

Acknowledgments: The authors acknowledge with thanks the Department of Mathematics and Applied Mathematics at the Sefako Makgatho Health Sciences University for making their facilities available for the research.

Conflicts of Interest: The authors declare no conflict of interest.

\section{References}

1. Glowinski, R.; Lions, J.L.; Trémoliéres, R. Numerical Analysis of Variational Inequalities; North-Holland: Amsterdam, The Netherlands, 1981.

2. Kinderlehrer, D.; Stampachia, G. An Introduction to Variational Inequalities and Their Applications; Society for Industrial and Applied Mathematics: Philadelphia, PA, USA, 2000.

3. Marcotte, P. Applications of Khobotov's algorithm to variational and network equilibrium problems. INFOR Inf. Syst. Oper. Res. 1991, 29, 255-270. [CrossRef]

4. Facchinei, F.; Pang, J. Finite-Dimensional Variational Inequalities and Complementary Problems; Springer: New York, NY, USA, 2003.

5. Reich, S.; Zalas, R. A modular string averaging procedure for solving the common fixed point problem for quasi-nonexpansive mappings in Hilbert space. Numer. Algorithm 2016, 72, 297-323. [CrossRef]

6. Censor, Y.; Gibali, A.; Reich, S.; Sabach, S. Common solutions to variational inequalities. Set Valued Var. Anal. 2012, 20, 229-247. [CrossRef]

7. Nadezhkina, N.; Takahashi, W. Strong convergence Theorem by a hybrid method for nonexpansive mappings and Lipschitz-continuous monotone mappings. SIAM Optim. 2006, 16, 1230-1241. [CrossRef]

8. Anh, P.N.; Phuong, N.X. A parallel extragradient-like projection method for unrelated variational inequalities and fixed point problem. J. Fixed Point Theory Appl. 2018, 20, 74. [CrossRef]

9. Anh, P.N.; Phuong, N.X. Linesearch methods for variational inequalities involving strict pseudocontractions. Optimization 2015, 64, 1841-1854. [CrossRef]

10. Cholamjiak, P.; Suantai, S.; Sunthrayuth, P. An explicit parallel algorithm for solving variational inclusion problem and fixed point problem in Banach spaces. Banach J. Math. Anal. 2020, 14, 20-40. [CrossRef]

11. Anh, P.K.; Hieu, D.V. Parallel hybrid methods for variational inequalities, equilibrium problems and common fixed point problems. Vietnam J. Math. 2016, 44, 351-374. [CrossRef]

12. Iiduka, H. A new iterative algorithm for the variational inequality problem over the fixed point set of a firmly nonexpansive mapping. Optimization 2010, 59, 873-885. [CrossRef]

13. Iiduka, H.; Yamada, I. A use of conjugate gradient direction for the convex optimization problem over the fixed point set of a nonexpansive mapping. SIAM J. Optim. 2009, 19, 1881-1893. [CrossRef]

14. Maingé, P.E. A hybrid extragradient-viscosity method for monotone operators and fixed point problems. SIAM J. Control Optim. 2008, 47, 1499-1515. [CrossRef]

15. Goldstein, A.A. Convex programming in Hilbert space. Bull. Am. Math. Soc. 1964, 70, 709-710. [CrossRef]

16. Korpelevich, G.M. The extragradient method for finding saddle points and other problems. Ekon. Mat. Metody 1976, 12, 747-756. (In Russian)

17. Vuong, P.T. On the weak convergence of the extragradient method for solving pseudo-monotone variational inequalities. J. Optim. Theory Appl. 2018, 176, 399-409. [CrossRef] [PubMed]

18. Censor, Y.; Gibali, A.; Reich, S. Extensions of Korpelevich's extragradient method for variational inequality problems in Euclidean space. Optimization 2012, 61, 119-1132. [CrossRef]

19. Censor, Y.; Gibali, A.; Reich, S. The subgradient extragradient method for solving variational inequalities in Hilbert space. J. Optim. Theory Appl. 2011, 148, 318-335. [CrossRef]

20. Ceng, L.C.; Hadjisavas, N.; Weng, N.C. Strong convergence Theorems by a hybrid extragradient-like approximation method for variational inequalities and fixed point problems. J. Glob. Optim. 2010, 46, 635-646. [CrossRef]

21. Jolaoso, L.O.; Aphane, M. Weak and strong convergence Bregman extragradient schemes for solving pseudo-monotone and non-Lipschitz variational inequalities. J. Ineq. Appl. 2020, 2020, 195. [CrossRef]

22. Jolaoso, L.O.; Aphane, M. A generalized viscosity inertial projection and contraction method for pseudomonotone variational inequality and fixed point problems. Mathematics 2020, 8, 2039. [CrossRef] 
23. Jolaoso, L.O.; Taiwo, A.; Alakoya, T.O.; Mewomo, O.T. A strong convergence Theorem for solving pseudo-monotone variational inequalities using projection methods in a reflexive Banach space. J. Optim. Theory Appl. 2020, 185, 744-766. [CrossRef]

24. He, B.S. A class of projection and contraction methods for monotone variational inequalities. Appl. Math. Optim. 1997, 35, 69-76. [CrossRef]

25. Solodov, M.V.; Svaiter, B.F. A new projection method for variational inequality problems. SIAM J. Control Optim. 1999, 37, 765-776. [CrossRef]

26. Migorski, S.; Fang, C.; Zeng, S. A new modified subgradient extragradient method for solving variational inequalities. Appl. Anal. 2019,1-10. [CrossRef]

27. Hieu, D.V.; Thong, D.V. New extragradient-like algorithms for strongly pseudomonotone variational inequalities. J. Glob. Optim. 2018, 70, 385-399. [CrossRef]

28. Dong, Q.-L.; Lu, Y.Y.; Yang, J. The extragradient algorithm with inertial effects for solving the variational inequality. Optimization 2016, 65, 2217-2226. [CrossRef]

29. Cholamjiak, P.; Thong, D.V.; Cho, Y.J. A novel inertial projection and contraction method for solving pseudomonotone variational inequality problem. Acta Appl. Math. 2020, 169, 217-245. [CrossRef]

30. Yamada, I. The hybrid steepest-descent method for variational inequalities problems over the intersection of the fixed point sets of nonexpansive mappings. In Inherently Parallel Algorithms in Feasibility and Optimization and Their Applications; Butnariu, D., Censor, Y., Reich, S., Eds.; North-Holland: Amsterdam, The Netherlands, 2001; pp. 473-504.

31. Hieu, D.V.; Son, D.X.; Anh, P.K.; Muu, L.D. A Two-Step Extragradient-Viscosity Method for Variational Inequalities and Fixed Point Problems. Acta Math. Vietnam. 2018, 2, 531-552. [CrossRef]

32. Anh, P.K.; Hieu, D.V. Parallel and sequential hybrid methods for a finite family of asymptotically quasi $\phi$-nonexpensive mappings. J. Appl. Math. Comput. 2015, 48, 241-263. [CrossRef]

33. Hieu, D.V. Parallel and cyclic hybrid subgradient extragradient methods for variational inequalities. Afr. Math. 2017, 28, 677-692. [CrossRef]

34. Rudin, W. Functional Analysis; McGraw-Hill Series in Higher Mathematics: New York, NY, USA, 1991.

35. Goebel, K.; Reich, S. Uniform Convexity, Hyperbolic Geometry, and Nonexpansive Mappings; Marcel Dekker: New York, NY, USA; Basel, Switzerland, 1984.

36. Cottle, R.W.; Yao, J.C. Pseudo-monotone complementarity problems in Hilbert space. J. Optim. Theory Appl. 1992, 75, 281-295. [CrossRef]

37. Maingé, P.E. Strong convergence of projected subgradient methods for nonsmooth and nonstrictly convex minimization. Set-Valued Anal. 2008, 16, 899-912. [CrossRef]

38. Marino, G.; Xu, H.K. Weak and strong convergence Theorems for strict pseudo-contraction in Hilbert spaces. J. Math. Anal. Appl. 2007, 329, 336-346. [CrossRef]

39. Xu, H.K. Iterative algorithms for nonlinear operators. J. Lond. Math. Soc. 2002, 66, 240-256. [CrossRef]

40. Suantai, S.; Peeyada, P.; Yambangwai, D.; Cholamjiak, W. A parallel-viscosity-type subgradient extragradientline method for finding the common solution of variational inequality problems applied to image restoration problems. Mathematics 2020, 8, 248. [CrossRef]

41. Anh, T.V.; Muu, L.D.; Son, D.X. Parallel algorithms for solving a class of variational inequalities over the common fixed points set of a finite family of demicontractive mappings. Numer. Funct. Anal. Optim. 2018, 39, 1477-1494. [CrossRef]

42. Hieu, D.V. An explicit parallel algorithm for variational inequalities. Bull. Malays. Math. Sci. Soc 2019, 42, 201-221. [CrossRef]

Publisher's Note: MDPI stays neutral with regard to jurisdictional claims in published maps and institutional affiliations.

(C) 2020 by the authors. Licensee MDPI, Basel, Switzerland. This article is an open access article distributed under the terms and conditions of the Creative Commons Attribution (CC BY) license (http://creativecommons.org/licenses/by/4.0/). 